\title{
Proton decay: flipped vs. unflipped SU(5)
}

\author{
John Ellis, ${ }^{a, b, c}$ Marcos A.G. Garcia, ${ }^{d}$ Natsumi Nagata, ${ }^{e}$ Dimitri V. Nanopoulos ${ }^{f, g, h}$ \\ and Keith A. Olive ${ }^{i}$ \\ ${ }^{a}$ Theoretical Particle Physics and Cosmology Group, Department of Physics, \\ King's College London, London WC2R 2LS, United Kingdom \\ ${ }^{b}$ Theoretical Physics Department, CERN, CH-1211 Geneva 23, Switzerland \\ ${ }^{c}$ National Institute of Chemical Physics and Biophysics, Rävala 10, 10143 Tallinn, Estonia \\ ${ }^{d}$ Instituto de Física Teórica (IFT) UAM-CSIC, Campus de Cantoblanco, 28049, Madrid, Spain \\ ${ }^{e}$ Department of Physics, University of Tokyo, Bunkyo-ku, Tokyo 113-0033, Japan \\ ${ }^{f}$ George P. and Cynthia W. Mitchell Institute for Fundamental Physics and Astronomy, \\ Texas A\&M University, College Station, TX 7r843, U.S.A. \\ ${ }^{g}$ Astroparticle Physics Group, Houston Advanced Research Center (HARC), \\ Mitchell Campus, Woodlands, TX 77381, U.S.A. \\ ${ }^{h}$ Academy of Athens, Division of Natural Sciences, Athens 10679, Greece \\ ${ }^{i}$ William I. Fine Theoretical Physics Institute, School of Physics and Astronomy, \\ University of Minnesota, Minneapolis, MN 55455, U.S.A. \\ E-mail: John.Ellis@cern.ch, marcosa.garcia@uam.es, \\ natsumi@hep-th.phys.s.u-tokyo.ac.jp, dimitri@physics.tamu.edu, \\ olive@umn.edu
}

AbSTRACT: We analyze nucleon decay modes in a no-scale supersymmetric flipped SU(5) GUT model, and contrast them with the predictions for proton decays via dimension-6 operators in a standard unflipped supersymmetric SU(5) GUT model. We find that these GUT models make very different predictions for the ratios $\Gamma\left(p \rightarrow \pi^{0} \mu^{+}\right) / \Gamma\left(p \rightarrow \pi^{0} e^{+}\right)$, $\Gamma\left(p \rightarrow \pi^{+} \bar{\nu}\right) / \Gamma\left(p \rightarrow \pi^{0} e^{+}\right), \Gamma\left(p \rightarrow K^{0} e^{+}\right) / \Gamma\left(p \rightarrow \pi^{0} e^{+}\right)$and $\Gamma\left(p \rightarrow K^{0} \mu^{+}\right) / \Gamma\left(p \rightarrow \pi^{0} \mu^{+}\right)$, and that predictions for the ratios $\Gamma\left(p \rightarrow \pi^{0} \mu^{+}\right) / \Gamma\left(p \rightarrow \pi^{0} e^{+}\right)$and $\Gamma\left(p \rightarrow \pi^{+} \bar{\nu}\right) / \Gamma\left(p \rightarrow \pi^{0} e^{+}\right)$ also differ in variants of the flipped $\mathrm{SU}(5)$ model with normal- or inverse-ordered light neutrino masses. Upcoming large neutrino experiments may have interesting opportunities to explore both GUT and flavour physics in proton and neutron decays.

KeYwords: Supersymmetry Phenomenology

ARXiv EPrint: 2003.03285 


\section{Contents}

1 Introduction 1

2 The no-scale flipped SU(5) model 3

$\begin{array}{lll}3 & \text { Nucleon decay in flipped SU(5) } & 6\end{array}$

4 Dimension-six proton decay in unflipped SU(5) 10

5 Comparison of proton decay rates in flipped and unflipped SU(5) 11

$5.1 \Gamma\left(p \rightarrow \pi^{0} \mu^{+}\right) / \Gamma\left(p \rightarrow \pi^{0} e^{+}\right) \quad 12$

$5.2 \sum_{i} \Gamma\left(p \rightarrow \pi^{+} \bar{\nu}_{i}\right) / \Gamma\left(p \rightarrow \pi^{0} e^{+}\right) \quad 15$

$5.3 \Gamma\left(p \rightarrow K^{0} e^{+}\right) / \Gamma\left(p \rightarrow \pi^{0} e^{+}\right) \quad 17$

$\begin{array}{lll}5.4 & \Gamma\left(p \rightarrow K^{0} \mu^{+}\right) / \Gamma\left(p \rightarrow \pi^{0} \mu^{+}\right) & 17\end{array}$

$\begin{array}{ll}5.5 p \rightarrow K^{+} \bar{\nu} & 17\end{array}$

$\begin{array}{llr}6 & \text { Discussion and prospects } & 18\end{array}$

\section{Introduction}

The advent of a new generation of high-mass underground neutrino detectors - JUNO [1], DUNE [2-4] and Hyper-Kamiokande [5] — will also open up new prospects for searches for proton (and neutron) decays into an array of channels with sensitivities an order of magnitude beyond current experiments. This motivates a re-evaluation of possible nucleon decay modes in different grand unified theories (GUTs), and analyses of specific signatures that may discriminate between the different models. A well-known example is the distinction that can be drawn between the minimal nonsupersymmetric SU(5) GUT [6] — in which the most characteristic proton decay mode is expected to be $p \rightarrow \pi^{0} e^{+}$induced by dimension- 6 operators - and the minimal supersymmetric SU(5) GUT $[7,8]$ — in which the dominant decay mode is expected to be $p \rightarrow K^{+} \bar{\nu}[9,10]$ induced by dimension- 5 operators $[11,12]$. The prospective sensitivities of the new generation of neutrino detectors to these decay modes has been documented [1-5], and the rate for $p \rightarrow K^{+} \bar{\nu}$ in the minimal supersymmetric SU(5) GUT has recently been re-evaluated, including an assessment of the uncertainties in the lifetime estimate [13].

As is well known, the difference between the dominant nucleon decays in the minimal supersymmetric and non-supersymmetric versions of $\mathrm{SU}(5)$ is linked to the difference between their respective decay mechanisms. Proton decay in minimal non-supersymmetric $\mathrm{SU}(5)$ is mediated by dimension- 6 operators $[14,15]$, whereas in minimal supersymmetric $\mathrm{SU}(5) p \rightarrow K^{+} \bar{\nu}$ is mediated by dimension-5 operators $[11,12]$. The rate for dimension- 5 
proton decay is high enough to put pressure on minimal supersymmetric $\mathrm{SU}(5)[16,17]$. This problem is mitigated by the higher sparticle masses [13, 18-29] now required by fruitless LHC searches [30-35], and dimension-5 proton decay may be suppressed by a discrete symmetry such as the hexality [36] appearing in some stringy extra-dimensional models with local grand unification. Nevertheless, the proton decay issue has added to the motivations for considering the supersymmetric flipped SU(5) GUT [37-43], in which an economical missing-partner mechanism [40, 44-46] suppresses dimension-5 proton decay. This model is also of interest because it can easily be accommodated within string theory [41-43, 47, 48], and a unified cosmological scenario for inflation, dark matter, neutrino masses and baryogenesis has been constructed [49-52] in the combined framework of flipped SU(5) and string-motivated [53] no-scale supergravity [54-56].

The dominant final states for proton decay in supersymmetric flipped $\mathrm{SU}(5)$ are not expected to contain strange particles, with many of the favoured decay modes expected to be similar to those in minimal supersymmetric SU(5), including $p \rightarrow \pi^{0} e^{+}$and $\pi^{+} \bar{\nu}$ [57]. It is therefore important to assemble a kit of diagnostic tools that the upcoming experiments can use to discriminate between the flipped and unflipped SU(5) GUT models. ${ }^{1}$ This issue has been discussed previously [68-74], and the purpose of this paper is to update the available diagnostic kit in the framework of the unified cosmological framework that we have proposed previously [49-52], stressing the connection between the flavour structure of nucleon decay operators and the pattern of mixing between neutrinos and their mass ordering.

We identify two primary proton decay signatures of the no-scale flipped SU(5) model [49-52] that may also cast light on the mass-ordering of light neutrinos. One signature is the ratio $\Gamma\left(p \rightarrow \pi^{0} \mu^{+}\right) / \Gamma\left(p \rightarrow \pi^{0} e^{+}\right)$, and the other is $\Gamma\left(p \rightarrow \pi^{+} \bar{\nu}\right) / \Gamma\left(p \rightarrow \pi^{0} e^{+}\right)^{2}$ In minimal $\mathrm{SU}(5)$ one expects $\Gamma\left(p \rightarrow \pi^{0} \mu^{+}\right) / \Gamma\left(p \rightarrow \pi^{0} e^{+}\right) \sim 0.008$, whereas this ratio is $\sim 0.1$ in flipped $\mathrm{SU}(5)$ with normally-ordered $(\mathrm{NO})$ light neutrinos and $\sim 23$ with inversely-ordered (IO) neutrinos. In the case of $\Gamma\left(p \rightarrow \pi^{+} \bar{\nu}\right) / \Gamma\left(p \rightarrow \pi^{0} e^{+}\right)$, the IO flipped SU(5) model predicts a ratio $\sim 95$ and the NO model predicts a ratio $\sim 3.2$, whereas the minimal $\mathrm{SU}(5)$ model allows values as low as 0.4. In addition to these headline signatures, we also find that the ratio $\Gamma\left(p \rightarrow K^{0} e^{+}\right) / \Gamma\left(p \rightarrow \pi^{0} e^{+}\right)$would be larger in flipped $\mathrm{SU}(5)$ than in minimal $\mathrm{SU}(5), \sim 0.02$ vs. $\sim 0.003$, whereas the ratio $\Gamma\left(p \rightarrow K^{0} \mu^{+}\right) / \Gamma\left(p \rightarrow \pi^{0} \mu^{+}\right) \sim 0.02$ in the flipped SU(5) model, as opposed to $\sim 17$ in minimal $\mathrm{SU}(5)$. It is clear therefore, that measurements of proton decay in more than one final state could discriminate between underlying GUT models, and we show that searches for neutron decays may also play an important role.

The outline of this paper is the following. In section 2 we review relevant features of the no-scale flipped SU(5) GUT model, and in section 3 we study proton (and some neutron) decay modes in this model, giving expressions in terms of the relevant hadronic matrix elements and discussing their uncertainties. The corresponding expressions in unflipped $\mathrm{SU}(5)$ are discussed in section 4. In section 5 we present predictions for ratios of proton decay rates in the flipped and unflipped SU(5) GUTs, and we review our conclusions and discuss future prospects in section 6 .

\footnotetext{
${ }^{1}$ See [58-67] for proposed diagnostic tools for other GUT models.

${ }^{2}$ Here and subsequently, the sum over the three light neutrino species is to be understood.
} 


\section{The no-scale flipped SU(5) model}

In the no-scale flipped SU(5) $\times \mathrm{U}(1)$ GUT model [37-43, 49-52], the three generations of the minimal supersymmetric extension of the Standard Model (MSSM) matter fields are embedded, together with three right-handed singlet neutrino chiral superfields, into three sets of $\mathbf{1 0}, \overline{\mathbf{5}}$, and $\mathbf{1}$ representations of SU(5), which we denote by $F_{i}, \bar{f}_{i}$ and $\ell_{i}^{c}$, respectively, where $i=1,2,3$ is the generation index. In units of $1 / \sqrt{40}$, the $\mathrm{U}(1)$ charges of the $F_{i}, \bar{f}_{i}$ and $\ell_{i}^{c}$ are $+1,-3$, and +5 , respectively. The assignments of the quantum numbers for the right-handed leptons, up- and down-type quarks are "flipped" with respect to the standard $\mathrm{SU}(5)$ assignments, giving the model its flippant name.

In addition to these matter fields, the minimal flipped $\mathrm{SU}(5)$ model contains a pair of $\mathbf{1 0}$ and $\overline{\mathbf{1 0}}$ Higgs fields, $H$ and $\bar{H}$, respectively, a pair of $\mathbf{5}$ and $\overline{\mathbf{5}}$ Higgs fields, $h$ and $\bar{h}$, respectively, and four singlet fields, $\phi_{a}(a=0, \ldots, 3)$. The vacuum expectation values (VEVs) of the $H$ and $\bar{H}$ fields break the $\mathrm{SU}(5) \times \mathrm{U}(1)$ gauge group down to the SM gauge group, and subsequently the VEVs of the doublet Higgs fields $H_{d}$ and $H_{u}$, which reside in $h$ and $\bar{h}$, respectively, break the $\mathrm{SU}(2)_{L} \times \mathrm{U}(1)_{Y}$ gauge symmetry down to the $\mathrm{U}(1)$ of electromagnetism.

The renormalizable superpotential in this model is given by

$$
\begin{aligned}
W= & \lambda_{1}^{i j} F_{i} F_{j} h+\lambda_{2}^{i j} F_{i} \bar{f}_{j} \bar{h}+\lambda_{3}^{i j} \bar{f}_{i} \ell_{j}^{c} h+\lambda_{4} H H h+\lambda_{5} \bar{H} \bar{H} \bar{h} \\
& +\lambda_{6}^{i a} F_{i} \bar{H} \phi_{a}+\lambda_{7}^{a} h \bar{h} \phi_{a}+\lambda_{8}^{a b c} \phi_{a} \phi_{b} \phi_{c}+\mu^{a b} \phi_{a} \phi_{b} .
\end{aligned}
$$

We assume here that the model possesses an approximate $\mathbb{Z}_{2}$ symmetry, under which only the $H$ field is odd while the rest of the fields are even. This symmetry is supposed to be violated by some Planck-scale suppressed operators, which prevent the formation of domain walls when the field $H$ acquires a VEV. This $\mathbb{Z}_{2}$ symmetry forbids some unwanted terms, such as $F_{i} H h$ and $\bar{f}_{i} H \bar{h}$, which would cause baryon/lepton-number violation as well as $R$-parity violation. The $\mathbb{Z}_{2}$ symmetry also forbids a vector-like mass term for $H$ and $\bar{H}$, which is advantageous for suppressing rapid proton decay induced by colour-triplet Higgs exchange.

We embed the flipped $\mathrm{SU}(5)$ model in minimal $N=1$ supergravity, which we assume to have a Kähler potential of no-scale form [56], as is motivated by the low-energy structure of string theory [53]. In this case the potential $V$ has an $F$ - and $D$-flat direction along a linear combination of the singlet components in $H$ and $\bar{H}$. These fields develop VEVs in this direction, as discussed in detail in ref. [50]. After $H$ and $\bar{H}$ acquire VEVs in this 'flaton' direction, the coloured components in these fields form vector-like multiplets with those in $h$ and $\bar{h}$ via the couplings $\lambda_{4}$ and $\lambda_{5}$ in (2.1). On the other hand, the electroweak doublets $H_{d}$ and $H_{u}$ in $h$ and $\bar{h}$ do not acquire masses from the flaton VEV - this is an economical realization of the missing-partner mechanism [40] that solves naturally the doublet-triplet splitting problem.

As discussed in detail in ref. [49], this model offers the possibility of successful Starobinsky-like [75] inflation, with one of the singlet fields, $\phi_{0}$, playing the role of the inflaton [76]. For $\mu^{00}=m_{s} / 2$ and $\lambda_{8}^{000}=-m_{s} /\left(3 \sqrt{3} M_{P}\right)$ in $(2.1)$ with the inflaton mass 
$m_{s} \simeq 3 \times 10^{13} \mathrm{GeV}$ and $M_{P} \equiv\left(8 \pi G_{N}\right)^{-1 / 2}$ the reduced Planck mass, the measured amplitude of the primordial power spectrum is successfully reproduced and the tensor-to-scalar ratio $r \simeq 3 \times 10^{-3}$, well within the range allowed by the Planck results and other data [77]. This prediction can be tested in future CMB experiments such as CMB-S4 [78, 79] and LiteBIRD [80]. The predicted value of the tilt in the scalar perturbation spectrum, $n_{s}$, is also within the range favoured by Planck and other data at the 68\% CL [77].

As seen in eq. (2.1), the inflaton $\phi_{0}$ can couple to the matter sector via the couplings $\lambda_{6}$ and $\lambda_{7}$. In ref. [49], two distinct cases, $\lambda_{6}^{i 0}=0$ (Scenario A) or $\lambda_{6}^{i 0} \neq 0$ (Scenario B), were studied. We focus on Scenario B in this work. In this scenario, one of the three singlet fields other than $\phi_{0}$, which we denote by $\phi_{3}$, does not have the $\lambda_{6}$ coupling; i.e., $\lambda_{6}^{i 3}=0$, whereas $\lambda_{6}^{i a} \neq 0$ for $i=1,2,3$ and $a=0,1,2$. We also assume $\lambda_{7}^{a}=0$ for $a=0,1,2$. To realize this scenario, we introduce a modified $R$-parity, under which the fields in this model transform as

$$
\begin{aligned}
F_{i}, \bar{f}_{i}, \ell_{i}^{c}, \phi_{0}, \phi_{1}, \phi_{2} & \rightarrow-F_{i},-\bar{f}_{i},-\ell_{i}^{c},-\phi_{0},-\phi_{1},-\phi_{2}, \\
H, \bar{H}, h, \bar{h}, \phi_{3} & \rightarrow H, \bar{H}, h, \bar{h}, \phi_{3} .
\end{aligned}
$$

We note that this modified $R$-parity is slightly violated by the coupling $\lambda_{8}^{000}$. Nevertheless, since this $R$-parity-violating effect is only very weakly transmitted to the matter sector, the lifetime of the lightest supersymmetric particle (LSP) is still much longer than the age of the Universe $[50,81]$, so the LSP can be a good dark matter candidate. We also note that the singlet $\phi_{3}$ can acquire a VEV without spontaneously breaking the modified $R$ parity. In this case, the coupling $\lambda_{7}^{3}$, which is allowed by the modified $R$-parity, generates an effective $\mu$ term for $h$ and $\bar{h}, \mu=\lambda_{7}^{3}\left\langle\phi_{3}\right\rangle$, just as in the next-to-minimal supersymmetric extension of the SM.

As discussed in detail in refs. [49-52], the $\lambda_{6}$ coupling in this model controls i) inflaton decays and reheating; ii) the gravitino production rate and therefore the non-thermal abundance of the LSP; iii) neutrino masses; and iv) the baryon asymmetry of the Universe via leptogenesis [82]. In particular, we showed in refs. [51, 52] by scanning over possible values of $\lambda_{6}$ that the observed values of neutrino masses, the dark matter abundance, and baryon asymmetry can be explained simultaneously, together with a soft supersymmetrybreaking scale in the multi- $\mathrm{TeV}$ range. In this paper, we study nucleon decays in the scenario developed in refs. [49-52].

Without loss of generality, we adopt the basis where $\lambda_{2}^{i j}$ and $\mu^{a b}$ are real and diagonal. In this case, the MSSM matter fields and right-handed neutrinos are embedded into the $\mathrm{SU}(5)$ representations as in $[68]:^{3}$

$$
\begin{aligned}
& F_{i} \ni\left\{Q_{i}, V_{i j} e^{-i \varphi_{j}} d_{j}^{c},\left(U_{\nu^{c}}\right)_{i j} \nu_{j}^{c}\right\}, \\
& \bar{f}_{i} \ni\left\{u_{i}^{c}, L_{j}\left(U_{l}\right)_{j i}\right\}, \\
& \ell_{i}^{c}=\left(U_{l^{c}}\right)_{i j} e_{j}^{c},
\end{aligned}
$$

\footnotetext{
${ }^{3}$ We use the basis in which $U_{u}=U_{u^{c}}=U_{\phi}=1$, where these matrices are as defined in ref. [68]. Moreover, we have removed the overall phase factor $U_{6}$ using the field redefinition of $F_{i}$ and $\bar{f}_{i}$ and expressed the diagonal phase matrix $U_{7}$ as $\left(U_{7}\right)_{i j}=e^{i \varphi_{i}} \delta_{i j}$.
} 
where the $V_{i j}$ are the Cabibbo-Kobayashi-Maskawa (CKM) matrix elements, $U_{\nu^{c}}, U_{l}$, and $U_{l^{c}}$ are unitary matrices, and the phase factors $\varphi_{i}$ satisfy the condition $\sum_{i} \varphi_{i}=0$ [68]. The components of the doublet fields $Q_{i}$ and $L_{i}$ are written as

$$
Q_{i}=\left(\begin{array}{c}
u_{i} \\
V_{i j} d_{j}
\end{array}\right), \quad L_{i}=\left(\begin{array}{c}
\left(U_{\mathrm{PMNS}}\right)_{i j} \nu_{j} \\
e_{i}
\end{array}\right)
$$

where $U_{\text {PMNS }}$ is the Pontecorvo-Maki-Nakagawa-Sakata (PMNS) matrix. ${ }^{4}$

The diagonal components of $\lambda_{2}^{i j}$ and $\mu^{a b}(a, b=0,1,2)$ are given by

$$
\lambda_{2} \simeq \frac{1}{\left\langle\bar{h}_{0}\right\rangle} \operatorname{diag}\left(m_{u}, m_{c}, m_{t}\right), \quad \mu=\frac{1}{2} \operatorname{diag}\left(m_{s}, \mu^{1}, \mu^{2}\right)
$$

where we take $m_{s}=3 \times 10^{13} \mathrm{GeV}$ (see above). In what follows we express these matrices as $\lambda_{2}^{i j}=\lambda_{2}^{i} \delta^{i j}$ and $\mu^{a b}=\mu^{a} \delta^{a b}$. The first equation in eq. (2.5) is only an approximate expression, since in general renormalization-group effects and threshold corrections cause $\lambda_{2}$ to deviate from the up-type Yukawa couplings at low energies. However, since these effects are at most $\mathcal{O}(10) \%$ and depend on the mass spectrum of the theory, we neglect them in the following analysis.

The neutrino/singlet-fermion mass matrix can be written as

$$
\mathcal{L}_{\text {mass }}=-\frac{1}{2}\left(\begin{array}{lll}
\nu_{i} & \nu_{j}^{c} & \tilde{\phi}_{a}
\end{array}\right)\left(\begin{array}{ccc}
0 & \lambda_{2}^{i j}\left\langle\bar{h}_{0}\right\rangle & 0 \\
\lambda_{2}^{i j}\left\langle\bar{h}_{0}\right\rangle & 0 & \lambda_{6}^{j a}\left\langle\tilde{\nu}_{\bar{H}}^{c}\right\rangle \\
0 & \lambda_{6}^{j a}\left\langle\tilde{\nu}_{\bar{H}}^{c}\right\rangle & \mu^{a}
\end{array}\right)\left(\begin{array}{c}
\nu_{i} \\
\nu_{j}^{c} \\
\tilde{\phi}_{a}
\end{array}\right)+\text { h.c. },
$$

where $i, j=1,2,3$ and $a=0,1,2$, and $\tilde{\phi}_{0}$ corresponds to the fermionic superpartner of the inflaton field $\phi_{0}$. The mass matrix of the right-handed neutrinos is then obtained from a first seesaw mechanism:

$$
\left(m_{\nu^{c}}\right)_{i j}=\sum_{a=0,1,2} \frac{\lambda_{6}^{i a} \lambda_{6}^{j a}}{\mu^{a}}\left\langle\tilde{\nu}_{\bar{H}}^{c}\right\rangle^{2},
$$

where $\left\langle\tilde{\nu}_{\bar{H}}^{c}\right\rangle$ denotes the VEV of the $F$ - and $D$-flat direction of the singlet components of $H$ and $\bar{H}$ : we take $\left\langle\tilde{\nu}_{\bar{H}}^{c}\right\rangle=10^{16} \mathrm{GeV}$ in the following analysis. We diagonalize the mass matrix in eq. (2.7) using a unitary matrix $U_{\nu^{c}}$ :

$$
m_{\nu^{c}}^{D}=U_{\nu^{c}}^{T} m_{\nu^{c}} U_{\nu^{c}} .
$$

The light neutrino mass matrix is then obtained through a second seesaw mechanism [84-92]:

$$
\left(m_{\nu}\right)_{i j}=\sum_{k} \frac{\lambda_{2}^{i} \lambda_{2}^{j}\left(U_{\nu^{c}}\right)_{i k}\left(U_{\nu^{c}}\right)_{j k}\left\langle\bar{h}_{0}\right\rangle^{2}}{\left(m_{\nu^{c}}^{D}\right)_{k}} .
$$

This mass matrix is diagonalised by a unitary matrix $U_{\nu}$, so that

$$
m_{\nu}^{D}=U_{\nu}^{*} m_{\nu} U_{\nu}^{\dagger}
$$

\footnotetext{
${ }^{4}$ We define the PMNS matrix as in the Review of Particle Physics (RPP) [83], and that $U_{\mathrm{PMNS}}=U_{\mathrm{MNS}}^{*}$ in the notation of ref. [68].
} 
We note that, given a matrix $\lambda_{6}^{i a}$, the eigenvalues of the $m_{\nu}$ and $m_{\nu^{c}}$ matrices, as well as the mixing matrices $U_{\nu^{c}}$ and $U_{\nu}$, are uniquely determined as functions of $\mu^{1}$ and $\mu^{2}$ via eqs. (2.7)-(2.9). The PMNS matrix is given by $U_{l}$ in eq. (2.3) and $U_{\nu}$ in eq. (2.10):

$$
U_{\mathrm{PMNS}}=U_{l}^{*} U_{\nu}^{T}
$$

Using the measured values of the PMNS matrix elements, we can use this relation to obtain $U_{l}$ from $U_{\nu}$. The matrix $U_{l}$ plays an important role in determining the partial decay widths of proton decay modes, as we will see in the subsequent section.

\section{$3 \quad$ Nucleon decay in flipped SU(5)}

We are now ready to discuss nucleon decay in our model. In view of the suppression of the dimension- 5 contribution mediated by coloured Higgs fields thanks to the missing-partner mechanism in the flipped SU(5) GUT [40], the main contribution to nucleon decay is due to exchanges of SU(5) gauge bosons. The relevant gauge interaction terms are

$$
K_{\text {gauge }}=\sqrt{2} g_{5}\left(-\epsilon_{\alpha \beta}\left(u_{a}^{c}\right)^{\dagger} X_{a}^{\alpha} U_{l}^{T} L^{\beta}+\epsilon^{a b c}\left(Q^{a \alpha}\right)^{\dagger} X_{b}^{\alpha} V P^{\dagger} d_{c}^{c}+\epsilon_{\alpha \beta}\left(\nu^{c}\right)^{\dagger} U_{\nu^{c}}^{\dagger} X_{a}^{\alpha} Q^{a \beta}+\text { h.c. }\right),
$$

where $g_{5}$ is the $\mathrm{SU}(5)$ gauge coupling constant, the $X_{a}^{\alpha}$ are the $\mathrm{SU}(5)$ gauge vector superfields, $P_{i j} \equiv e^{i \varphi_{i}} \delta_{i j}, \alpha, \beta$ are $\mathrm{SU}(2)_{L}$ indices, and $a, b, c$ are $\mathrm{SU}(3)_{C}$ indices.

Below the GUT scale, the effects of $\mathrm{SU}(5)$ gauge boson exchanges are in general described by the dimension-six effective operators

$$
\mathcal{L}_{6}^{\mathrm{eff}}=C_{6(1)}^{i j k l} \mathcal{O}_{i j k l}^{6(1)}+C_{6(2)}^{i j k l} \mathcal{O}_{i j k l}^{6(2)}
$$

where

$$
\begin{aligned}
\mathcal{O}_{i j k l}^{6(1)} & =\int d^{2} \theta d^{2} \bar{\theta} \epsilon_{a b c} \epsilon_{\alpha \beta}\left(u_{i}^{c \dagger}\right)^{a}\left(d_{j}^{c \dagger}\right)^{b} e^{-\frac{2}{3} g^{\prime} B}\left(e^{2 g_{3} G} Q_{k}^{\alpha}\right)^{c} L_{l}^{\beta}, \\
\mathcal{O}_{i j k l}^{6(2)} & =\int d^{2} \theta d^{2} \bar{\theta} \epsilon_{a b c} \epsilon_{\alpha \beta} Q_{i}^{a \alpha} Q_{j}^{b \beta} e^{\frac{2}{3} g^{\prime} B}\left(e^{-2 g_{3} G} u_{k}^{c \dagger}\right)^{c} e_{l}^{c \dagger},
\end{aligned}
$$

with $G$ and $B$ the $\mathrm{SU}(3)_{C}$ and $\mathrm{U}(1)_{Y}$ gauge vector superfields, respectively, and $g_{3}$ and $g^{\prime}$ the corresponding gauge couplings. In the unflipped SU(5) GUT both of the Wilson coefficients $C_{6(1,2)}^{i j k l}$ are non-zero, but in flipped $\mathrm{SU}(5)$ only $C_{6(1)}^{i j k l}$ is non-zero, and is given by ${ }^{5}$

$$
C_{6(1)}^{i j k l}=\frac{g_{5}^{2}}{M_{X}^{2}}\left(U_{l}\right)_{l i} V_{k j}^{*} e^{i \varphi_{j}}
$$

where $M_{X}$ is the SU(5) gauge boson mass. The Wilson coefficients are run down to low energy scales using the renormalisation group equations. The renormalisation factors for

\footnotetext{
${ }^{5}$ However, although $C_{6(2)}^{i j k l}$ vanishes in flipped SU(5), we retain it in the following formulae so that it can also be used for the unflipped case.
} 


\begin{tabular}{|lc|}
\hline Matrix element & Value $\left[\mathrm{GeV}^{2}\right]$ \\
\hline$\left\langle\pi^{0}\left|(u d)_{R} u_{L}\right| p\right\rangle_{e}$ & $-0.131(4)(13)$ \\
$\left\langle\pi^{0}\left|(u d)_{R} u_{L}\right| p\right\rangle_{\mu}$ & $-0.118(3)(12)$ \\
$\left\langle\pi^{+}\left|(u d)_{R} d_{L}\right| p\right\rangle$ & $-0.186(6)(18)$ \\
$\left\langle K^{0}\left|(u s)_{R} u_{L}\right| p\right\rangle_{e}$ & $0.103(3)(11)$ \\
$\left\langle K^{0}\left|(u s)_{R} u_{L}\right| p\right\rangle_{\mu}$ & $0.099(2)(10)$ \\
$\left\langle K^{+}\left|(u s)_{R} d_{L}\right| p\right\rangle$ & $-0.049(2)(5)$ \\
$\left\langle K^{+}\left|(u d)_{R} s_{L}\right| p\right\rangle$ & $-0.134(4)(14)$ \\
\hline
\end{tabular}

Table 1. Hadronic matrix elements used in our analysis, which are taken from ref. [97]. The statistical and systematic uncertainties are indicated by $(\ldots)(\ldots)$. The subscripts $e$ and $\mu$ indicate that the matrix elements are evaluated at the corresponding lepton kinematic points.

$C_{6(n)}^{i j k l}(n=1,2)$ between the GUT scale and the electroweak scale, $A_{S_{n}}$, are evaluated at the one-loop level ${ }^{6}$ as $[94,95]$ :

$$
\begin{aligned}
A_{S_{1}}= & {\left[\frac{\alpha_{3}\left(\mu_{\mathrm{SUSY}}\right)}{\alpha_{3}\left(\mu_{\mathrm{GUT}}\right)}\right]^{\frac{4}{9}}\left[\frac{\alpha_{2}\left(\mu_{\mathrm{SUSY}}\right)}{\alpha_{2}\left(\mu_{\mathrm{GUT}}\right)}\right]^{-\frac{3}{2}}\left[\frac{\alpha_{1}\left(\mu_{\mathrm{SUSY}}\right)}{\alpha_{1}\left(\mu_{\mathrm{GUT}}\right)}\right]^{-\frac{1}{18}} } \\
& \times\left[\frac{\alpha_{3}\left(m_{Z}\right)}{\alpha_{3}\left(\mu_{\mathrm{SUSY}}\right)}\right]^{\frac{2}{7}}\left[\frac{\alpha_{2}\left(m_{Z}\right)}{\alpha_{2}\left(\mu_{\mathrm{SUSY}}\right)}\right]^{\frac{27}{38}}\left[\frac{\alpha_{1}\left(m_{Z}\right)}{\alpha_{1}\left(\mu_{\mathrm{SUSY}}\right)}\right]^{-\frac{11}{82}}, \\
A_{S_{2}}= & {\left[\frac{\alpha_{3}\left(\mu_{\mathrm{SUSY}}\right)}{\alpha_{3}\left(\mu_{\mathrm{GUT}}\right)}\right]^{\frac{4}{9}}\left[\frac{\alpha_{2}\left(\mu_{\mathrm{SUSY}}\right)}{\alpha_{2}\left(\mu_{\mathrm{GUT}}\right)}\right]^{-\frac{3}{2}}\left[\frac{\alpha_{1}\left(\mu_{\mathrm{SUSY}}\right)}{\alpha_{1}\left(\mu_{\mathrm{GUT}}\right)}\right]^{-\frac{23}{198}} } \\
& \times\left[\frac{\alpha_{3}\left(m_{Z}\right)}{\alpha_{3}\left(\mu_{\mathrm{SUSY}}\right)}\right]^{\frac{2}{7}}\left[\frac{\alpha_{2}\left(m_{Z}\right)}{\alpha_{2}\left(\mu_{\mathrm{SUSY}}\right)}\right]^{\frac{27}{38}}\left[\frac{\alpha_{1}\left(m_{Z}\right)}{\alpha_{1}\left(\mu_{\mathrm{SUSY}}\right)}\right]^{-\frac{23}{82}},
\end{aligned}
$$

where $m_{Z}, \mu_{\mathrm{SUSY}}$, and $\mu_{\mathrm{GUT}}$ denote the $Z$-boson mass, the SUSY scale and the GUT scale, respectively, and $\alpha_{A} \equiv g_{A}^{2} /(4 \pi)$ with $g_{A}(A=1,2,3)$ the gauge coupling constants of the SM gauge groups. We give the electroweak-scale matching conditions for each decay mode in what follows. Below the electroweak scale, we take into account the perturbative QCD renormalization factor, which is computed in ref. [96] at the two-loop level: $A_{L}=1.247$. We then calculate the partial decay widths of various proton decay modes by using the corresponding hadronic matrix elements, for which we use the results obtained from the QCD lattice simulation performed in ref. [97]. The relevant hadronic matrix elements are listed in table 1.

In the following we summarise the partial decay widths for the proton decay modes that we discuss in this paper, as well as two relevant neutron decay modes. ${ }^{7}$

$p \rightarrow \pi^{0} e^{+}$. The relevant effective operators below the electroweak scale are

$$
\mathcal{L}\left(p \rightarrow \pi^{0} l_{i}^{+}\right)=C_{R L}\left(u d u l_{i}\right)\left[\epsilon_{a b c}\left(u_{R}^{a} d_{R}^{b}\right)\left(u_{L}^{c} l_{L i}\right)\right]+C_{L R}\left(u d u l_{i}\right)\left[\epsilon_{a b c}\left(u_{L}^{a} d_{L}^{b}\right)\left(u_{R}^{c} l_{R i}\right)\right],
$$

\footnotetext{
${ }^{6}$ The two-loop RGEs for these coefficients above the SUSY-breaking scale are given in ref. [93].

${ }^{7}$ We note that these partial decay widths do not depend on the phases $\varphi_{i}$.
} 
where

$$
\begin{aligned}
& C_{R L}\left(u d u l_{i}\right)=C_{6(1)}^{111 i}\left(m_{Z}\right), \\
& C_{L R}\left(u d u l_{i}\right)=V_{j 1}\left[C_{6(2)}^{1 j 1 i}\left(m_{Z}\right)+C_{6(2)}^{j 11 i}\left(m_{Z}\right)\right] .
\end{aligned}
$$

Note that, since $C_{6(2)}^{i j k l}=0$ in flipped SU(5), the second term in eq. (3.7) vanishes for this model. The partial decay width can be expressed as follows in terms of these coefficients at the hadronic scale:

$$
\Gamma\left(p \rightarrow \pi^{0} l_{i}^{+}\right)=\frac{m_{p}}{32 \pi}\left(1-\frac{m_{\pi}^{2}}{m_{p}^{2}}\right)^{2}\left[\left|\mathcal{A}_{L}\left(p \rightarrow \pi^{0} l_{i}^{+}\right)\right|^{2}+\left|\mathcal{A}_{R}\left(p \rightarrow \pi^{0} l_{i}^{+}\right)\right|^{2}\right],
$$

where

$$
\begin{aligned}
& \mathcal{A}_{L}\left(p \rightarrow \pi^{0} l_{i}^{+}\right)=C_{R L}\left(u d u l_{i}\right)\left\langle\pi^{0}\left|(u d)_{R} u_{L}\right| p\right\rangle, \\
& \mathcal{A}_{R}\left(p \rightarrow \pi^{0} l_{i}^{+}\right)=C_{L R}\left(u d u l_{i}\right)\left\langle\pi^{0}\left|(u d)_{R} u_{L}\right| p\right\rangle .
\end{aligned}
$$

Setting $i=1$ in eq. (3.9), we obtain

$$
\Gamma\left(p \rightarrow \pi^{0} e^{+}\right)_{\text {flipped }}=\frac{g_{5}^{4} m_{p}\left|V_{u d}\right|^{2}\left|\left(U_{l}\right)_{11}\right|^{2}}{32 \pi M_{X}^{4}}\left(1-\frac{m_{\pi}^{2}}{m_{p}^{2}}\right)^{2} A_{L}^{2} A_{S_{1}}^{2}\left(\left\langle\pi^{0}\left|(u d)_{R} u_{L}\right| p\right\rangle_{e}\right)^{2},
$$

where $m_{p}$ and $m_{\pi}$ denote the masses of the proton and pion, respectively, and here and subsequently the subscript on the hadronic matrix element indicates that it is evaluated at the corresponding lepton kinematic point.

From eq. (3.11), we can readily compute the partial lifetime of the $p \rightarrow \pi^{0} e^{+}$mode as

$$
\tau\left(p \rightarrow \pi^{0} e^{+}\right)_{\text {flipped }} \simeq 7.9 \times 10^{35} \times\left|\left(U_{l}\right)_{11}\right|^{-2}\left(\frac{M_{X}}{10^{16} \mathrm{GeV}}\right)^{4}\left(\frac{0.0378}{\alpha_{5}}\right)^{2} \mathrm{yrs} .
$$

We note that this tends to be longer than the lifetime predicted in unflipped $\mathrm{SU}(5)$ by a factor (see also eq. (4.2))

$$
\frac{\tau\left(p \rightarrow \pi^{0} e^{+}\right)_{\text {flipped }}}{\tau\left(p \rightarrow \pi^{0} e^{+}\right)_{\text {unflipped }}} \simeq \frac{A_{S_{1}}^{2}+\left(1+\left|V_{u d}\right|^{2}\right)^{2} A_{S_{2}}^{2}}{A_{S_{1}}^{2}\left|\left(U_{l}\right)_{11}\right|^{2}} \simeq \frac{4.8}{\left|\left(U_{l}\right)_{11}\right|^{2}},
$$

as found in refs. $[17,57,68,70] .^{8}$

$\boldsymbol{p} \rightarrow \boldsymbol{\pi}^{\mathbf{0}} \boldsymbol{\mu}^{+}$. By using the effective Lagrangian in eq. (3.7) and the rate in eq. (3.9) for $i=2$, we have

$$
\Gamma\left(p \rightarrow \pi^{0} \mu^{+}\right)_{\text {flipped }}=\frac{g_{5}^{4} m_{p}\left|V_{u d}\right|^{2}\left|\left(U_{l}\right)_{21}\right|^{2}}{32 \pi M_{X}^{4}}\left(1-\frac{m_{\pi}^{2}}{m_{p}^{2}}\right)^{2} A_{L}^{2} A_{S_{1}}^{2}\left(\left\langle\pi^{0}\left|(u d)_{R} u_{L}\right| p\right\rangle_{\mu}\right)^{2},
$$

and the partial lifetime of the $p \rightarrow \pi^{0} \mu^{+}$mode is

$$
\tau\left(p \rightarrow \pi^{0} \mu^{+}\right)_{\text {flipped }} \simeq 9.7 \times 10^{35} \times\left|\left(U_{l}\right)_{21}\right|^{-2}\left(\frac{M_{X}}{10^{16} \mathrm{GeV}}\right)^{4}\left(\frac{0.0378}{\alpha_{5}}\right)^{2} \mathrm{yrs} .
$$

\footnotetext{
${ }^{8}$ Values of $\left(U_{l}\right)_{11}$ in specific flipped SU(5) GUT scenarios are discussed later: see eq. (5.9).
} 
$\boldsymbol{n} \rightarrow \boldsymbol{\pi}^{-} \boldsymbol{l}^{+}$. We note in passing that the rates of neutron decay modes that include a charged lepton can be obtained from $\Gamma\left(p \rightarrow \pi^{0} l_{i}^{+}\right)$through $\mathrm{SU}(2)$ isospin relations:

$$
\Gamma\left(n \rightarrow \pi^{-} l_{i}^{+}\right)=2 \Gamma\left(p \rightarrow \pi^{0} l_{i}^{+}\right),
$$

which applies to both the flipped and unflipped SU(5) models.

$\boldsymbol{p} \rightarrow \boldsymbol{\pi}^{+} \overline{\boldsymbol{\nu}}_{\boldsymbol{i}} . \quad$ The relevant effective Lagrangian term in this case is

$$
\mathcal{L}\left(p \rightarrow \pi^{+} \bar{\nu}_{i}\right)=C_{R L}\left(u d d \nu_{i}\right)\left[\epsilon_{a b c}\left(u_{R}^{a} d_{R}^{b}\right)\left(d_{L}^{c} \nu_{L i}\right)\right],
$$

with the following matching condition at the electroweak scale

$$
C_{R L}\left(u d d \nu_{i}\right)=-V_{j 1} C_{6(1)}^{11 j i}\left(m_{Z}\right) .
$$

The partial decay width is then computed as

$$
\Gamma\left(p \rightarrow \pi^{+} \bar{\nu}_{i}\right)=\frac{m_{p}}{32 \pi}\left(1-\frac{m_{\pi}^{2}}{m_{p}^{2}}\right)^{2}\left|\mathcal{A}\left(p \rightarrow \pi^{+} \bar{\nu}_{i}\right)\right|^{2},
$$

with

$$
\mathcal{A}\left(p \rightarrow \pi^{+} \bar{\nu}_{i}\right)=C_{R L}\left(u d d \nu_{i}\right)\left\langle\pi^{+}\left|(u d)_{R} d_{L}\right| p\right\rangle .
$$

We then have

$$
\Gamma\left(p \rightarrow \pi^{+} \bar{\nu}_{i}\right)_{\text {flipped }}=\frac{g_{5}^{4} m_{p}\left|\left(U_{l}\right)_{i 1}\right|^{2}}{32 \pi M_{X}^{4}}\left(1-\frac{m_{\pi}^{2}}{m_{p}^{2}}\right)^{2} A_{L}^{2} A_{S_{1}}^{2}\left(\left\langle\pi^{+}\left|(u d)_{R} d_{L}\right| p\right\rangle\right)^{2} .
$$

$\boldsymbol{n} \rightarrow \boldsymbol{\pi}^{\mathbf{0}} \overline{\boldsymbol{\nu}}_{\boldsymbol{i}}$. There is a relation between the partial decay widths for $n \rightarrow \pi^{0} \bar{\nu}_{i}$ and those of $p \rightarrow \pi^{+} \bar{\nu}_{i}$ given by isospin:

$$
\Gamma\left(n \rightarrow \pi^{0} \bar{\nu}_{i}\right)=\frac{1}{2} \Gamma\left(p \rightarrow \pi^{+} \bar{\nu}_{i}\right),
$$

which applies to both the flipped and unflipped SU(5) models.

$\boldsymbol{p} \rightarrow \boldsymbol{K}^{\mathbf{0}} \boldsymbol{e}^{+}$. The effective interactions in this case are given by

$$
\mathcal{L}\left(p \rightarrow K^{0} l_{i}^{+}\right)=C_{R L}\left(u s u l_{i}\right)\left[\epsilon_{a b c}\left(u_{R}^{a} s_{R}^{b}\right)\left(u_{L}^{c} l_{L i}\right)\right]+C_{L R}\left(u s u l_{i}\right)\left[\epsilon_{a b c}\left(u_{L}^{a} s_{L}^{b}\right)\left(u_{R}^{c} l_{R i}\right)\right],
$$

with

$$
\begin{aligned}
& C_{R L}\left(u_{s u l}\right)=C_{6(1)}^{121 i}\left(m_{Z}\right), \\
& C_{L R}\left(u_{s u l_{i}}\right)=V_{j 2}\left[C_{6(2)}^{1 j 1 i}\left(m_{Z}\right)+C_{6(2)}^{j 11 i}\left(m_{Z}\right)\right] .
\end{aligned}
$$

We then obtain the partial decay width

$$
\Gamma\left(p \rightarrow K^{0} l_{i}^{+}\right)=\frac{m_{p}}{32 \pi}\left(1-\frac{m_{K}^{2}}{m_{p}^{2}}\right)^{2}\left[\left|\mathcal{A}_{L}\left(p \rightarrow K^{0} l_{i}^{+}\right)\right|^{2}+\left|\mathcal{A}_{R}\left(p \rightarrow K^{0} l_{i}^{+}\right)\right|^{2}\right],
$$

where $m_{K}$ is the kaon mass and

$$
\begin{aligned}
& \mathcal{A}_{L}\left(p \rightarrow K^{0} l_{i}^{+}\right)=C_{R L}\left(\text { usul }_{i}\right)\left\langle K^{0}\left|(u s)_{R} u_{L}\right| p\right\rangle, \\
& \mathcal{A}_{R}\left(p \rightarrow K^{0} l_{i}^{+}\right)=C_{L R}\left(\text { usul }_{i}\right)\left\langle K^{0}\left|(u s)_{R} u_{L}\right| p\right\rangle .
\end{aligned}
$$

In particular, for $i=1$, we have

$$
\Gamma\left(p \rightarrow K^{0} e^{+}\right)_{\text {flipped }}=\frac{g_{5}^{4} m_{p}\left|V_{u s}\right|^{2}\left|\left(U_{l}\right)_{11}\right|^{2}}{32 \pi M_{X}^{4}}\left(1-\frac{m_{K}^{2}}{m_{p}^{2}}\right)^{2} A_{L}^{2} A_{S_{1}}^{2}\left(\left\langle K^{0}\left|(u s)_{R} u_{L}\right| p\right\rangle_{e}\right)^{2} .
$$


$\boldsymbol{p} \rightarrow \boldsymbol{K}^{\mathbf{0}} \boldsymbol{\mu}^{+}$. With $i=2$ in eq. (3.25), we have

$$
\Gamma\left(p \rightarrow K^{0} \mu^{+}\right)_{\text {flipped }}=\frac{g_{5}^{4} m_{p}\left|V_{u s}\right|^{2}\left|\left(U_{l}\right)_{21}\right|^{2}}{32 \pi M_{X}^{4}}\left(1-\frac{m_{K}^{2}}{m_{p}^{2}}\right)^{2} A_{L}^{2} A_{S_{1}}^{2}\left(\left\langle K^{0}\left|(u s)_{R} u_{L}\right| p\right\rangle_{\mu}\right)^{2} .
$$

$\boldsymbol{p} \rightarrow \boldsymbol{K}^{+} \overline{\boldsymbol{\nu}}_{\boldsymbol{i}}$. The low-energy effective interactions for this decay mode is given by

$$
\mathcal{L}\left(p \rightarrow K^{+} \bar{\nu}_{i}\right)=C_{R L}\left(u s d \nu_{i}\right)\left[\epsilon_{a b c}\left(u_{R}^{a} s_{R}^{b}\right)\left(d_{L}^{c} \nu_{i}\right)\right]+C_{R L}\left(u d s \nu_{i}\right)\left[\epsilon_{a b c}\left(u_{R}^{a} d_{R}^{b}\right)\left(s_{L}^{c} \nu_{i}\right)\right],
$$

with

$$
\begin{aligned}
& C_{R L}\left(u s d \nu_{i}\right)=-V_{j 1} C_{6(1)}^{12 j i}\left(m_{Z}\right), \\
& C_{R L}\left(u d s \nu_{i}\right)=-V_{j 2} C_{6(1)}^{11 j i}\left(m_{Z}\right) .
\end{aligned}
$$

We note that the unitarity of the CKM matrix leads to

$$
V_{j 1} C_{6(1)}^{12 j i}=V_{j 2} C_{6(1)}^{11 j i}=0,
$$

in the case of flipped SU(5). As a result, we have

$$
\Gamma\left(p \rightarrow K^{+} \bar{\nu}_{i}\right)=0,
$$

as found in ref. [68].

\section{Dimension-six proton decay in unflipped SU(5)}

In this section we review briefly the proton decay calculation in unflipped $\mathrm{SU}(5)$, assuming that proton decay is dominantly induced by dimension-6 SU(5) gauge boson exchange, i.e., that the dimension-5 contribution of colour-triplet Higgs exchange is negligible. This assumption is valid, e.g., when the sfermion masses are sufficiently large, i.e., $\gtrsim 100 \mathrm{TeV}[13$, 18-24] or if a suitable missing-partner mechanism is invoked [44-46]. For more detailed discussions of the calculation of proton decay induced by $\mathrm{SU}(5)$ gauge boson exchange in unflipped SU(5), see refs. [13, 20, 24, 98, 99].

In unflipped SU(5), the Wilson coefficients of the effective operators in eq. (3.2) are given by

$$
\begin{aligned}
& C_{6(1)}^{i j k l}=-\frac{g_{5}^{2}}{M_{X}^{2}} e^{i \varphi_{i}} \delta^{i k} \delta^{j l}, \\
& C_{6(2)}^{i j k l}=-\frac{g_{5}^{2}}{M_{X}^{2}} e^{i \varphi_{i}} \delta^{i k}\left(V^{*}\right)^{j l} .
\end{aligned}
$$

The rest of the calculation is exactly the same as before, so we just summarize the resultant expression for each partial decay width.

$p \rightarrow \pi^{0} e^{+}$.

$$
\Gamma\left(p \rightarrow \pi^{0} e^{+}\right)=\frac{g_{5}^{4} m_{p}}{32 \pi M_{X}^{4}}\left(1-\frac{m_{\pi}^{2}}{m_{p}^{2}}\right)^{2} A_{L}^{2}\left(\left\langle\pi^{0}\left|(u d)_{R} u_{L}\right| p\right\rangle_{e}\right)^{2}\left[A_{S_{1}}^{2}+\left(1+\left|V_{u d}\right|^{2}\right)^{2} A_{S_{2}}^{2}\right] .
$$


$p \rightarrow \pi^{0} \mu^{+}$

$\Gamma\left(p \rightarrow \pi^{0} \mu^{+}\right)=\frac{g_{5}^{4} m_{p}}{32 \pi M_{X}^{4}}\left(1-\frac{m_{\pi}^{2}}{m_{p}^{2}}\right)^{2} A_{L}^{2} A_{S_{2}}^{2}\left(\left\langle\pi^{0}\left|(u d)_{R} u_{L}\right| p\right\rangle_{\mu}\right)^{2}\left[\left|V_{u d} V_{u s}^{*}\right|^{2}\right]$.

$p \rightarrow \pi^{+} \bar{\nu}$

$$
\Gamma\left(p \rightarrow \pi^{+} \bar{\nu}_{i}\right)=\frac{g_{5}^{4} m_{p}\left|V_{u d}\right|^{2}}{32 \pi M_{X}^{4}}\left(1-\frac{m_{\pi}^{2}}{m_{p}^{2}}\right)^{2} A_{L}^{2} A_{S_{1}}^{2} \delta^{1 i}\left(\left\langle\pi^{+}\left|(u d)_{R} d_{L}\right| p\right\rangle\right)^{2} .
$$

$p \rightarrow K^{0} e^{+}$

$$
\Gamma\left(p \rightarrow K^{0} e^{+}\right)=\frac{g_{5}^{4} m_{p}}{32 \pi M_{X}^{4}}\left(1-\frac{m_{K}^{2}}{m_{p}^{2}}\right)^{2} A_{L}^{2} A_{S_{2}}^{2}\left(\left\langle K^{0}\left|(u s)_{R} u_{L}\right| p\right\rangle_{e}\right)^{2}\left[\left|V_{u d} V_{u s}^{*}\right|^{2}\right] .
$$

$p \rightarrow K^{0} \mu^{+}$

$$
\Gamma\left(p \rightarrow K^{0} \mu^{+}\right)=\frac{g_{5}^{4} m_{p}}{32 \pi M_{X}^{4}}\left(1-\frac{m_{K}^{2}}{m_{p}^{2}}\right)^{2} A_{L}^{2}\left(\left\langle K^{0}\left|(u s)_{R} u_{L}\right| p\right\rangle_{\mu}\right)^{2}\left[A_{S_{1}}^{2}+\left(1+\left|V_{u s}\right|^{2}\right)^{2} A_{S_{2}}^{2}\right] .
$$

$p \rightarrow K^{+} \bar{\nu}$

$$
\begin{aligned}
\Gamma\left(p \rightarrow K^{+} \bar{\nu}_{i}\right)= & \frac{g_{5}^{4} m_{p}}{32 \pi M_{X}^{4}}\left(1-\frac{m_{K}^{2}}{m_{p}^{2}}\right)^{2} A_{L}^{2} A_{S_{1}}^{2} \\
& \times\left[\delta^{1 i}\left|V_{u s}\right|^{2}\left(\left\langle K^{+}\left|(u d)_{R} s_{L}\right| p\right\rangle\right)^{2}+\delta^{2 i}\left|V_{u d}\right|^{2}\left(\left\langle K^{+}\left|(u s)_{R} d_{L}\right| p\right\rangle\right)^{2}\right] .
\end{aligned}
$$

\section{Comparison of proton decay rates in flipped and unflipped SU(5)}

As we now discuss, the predictions for proton decay branching fractions in the flipped SU(5) GUT model are different from those generated by dimension- 6 operators in the standard unflipped SU(5) GUT, ${ }^{9}$ which may enable future experiments to distinguish these two GUT scenarios. To this end, we focus on the following five quantities and compare the predictions for them in flipped and unflipped SU(5) GUTs:

i) $\Gamma\left(p \rightarrow \pi^{0} \mu^{+}\right) / \Gamma\left(p \rightarrow \pi^{0} e^{+}\right)$,

ii) $\sum_{i} \Gamma\left(p \rightarrow \pi^{+} \bar{\nu}_{i}\right) / \Gamma\left(p \rightarrow \pi^{0} e^{+}\right)$,

iii) $\Gamma\left(p \rightarrow K^{0} e^{+}\right) / \Gamma\left(p \rightarrow \pi^{0} e^{+}\right)$,

iv) $\Gamma\left(p \rightarrow K^{0} \mu^{+}\right) / \Gamma\left(p \rightarrow \pi^{0} \mu^{+}\right)$,

v) $p \rightarrow K^{+} \bar{\nu}$.

\footnotetext{
${ }^{9}$ We assume here that the contributions of dimension- 5 operators are suppressed, either by large sparticle and/or triplet Higgs masses, or by some missing-partner mechanism.
} 


\section{$5.1 \Gamma\left(p \rightarrow \pi^{0} \mu^{+}\right) / \Gamma\left(p \rightarrow \pi^{0} e^{+}\right)$}

From eqs. (3.11) and (3.14), we find that this ratio in the flipped $\mathrm{SU}(5)$ is given by

$$
\frac{\Gamma\left(p \rightarrow \pi^{0} \mu^{+}\right)_{\text {flipped }}}{\Gamma\left(p \rightarrow \pi^{0} e^{+}\right)_{\text {flipped }}}=\frac{\left(\left\langle\pi^{0}\left|(u d)_{R} u_{L}\right| p\right\rangle_{\mu}\right)^{2}\left|\left(U_{l}\right)_{21}\right|^{2}}{\left(\left\langle\pi^{0}\left|(u d)_{R} u_{L}\right| p\right\rangle_{e}\right)^{2}\left|\left(U_{l}\right)_{11}\right|^{2}} .
$$

We see that this ratio depends on the unitary matrix $U_{l}$, which is determined from $U_{\nu}$ and the PMNS matrix $U_{\text {PMNS }}$ via eq. (2.11). We also note that by taking the ratio between the two partial decay widths $\Gamma\left(p \rightarrow \pi^{0} \mu^{+}\right)$and $\Gamma\left(p \rightarrow \pi^{0} e^{+}\right)$, many of the factors in these quantities such as the $\mathrm{SU}(5)$ gauge boson mass, $M_{X}$, the $\mathrm{SU}(5)$ gauge coupling constant, $g_{5}$, and the renormalization factors, $A_{L}$ and $A_{S_{1}}$, are cancelled, which makes the prediction for this ratio rather robust.

In unflipped SU(5), on the other hand, we obtain (see eqs. (4.2) and (4.3)):

$$
\frac{\Gamma\left(p \rightarrow \pi^{0} \mu^{+}\right)_{\text {unflipped }}}{\Gamma\left(p \rightarrow \pi^{0} e^{+}\right)_{\text {unflipped }}}=\frac{\left(\left\langle\pi^{0}\left|(u d)_{R} u_{L}\right| p\right\rangle_{\mu}\right)^{2}}{\left(\left\langle\pi^{0}\left|(u d)_{R} u_{L}\right| p\right\rangle_{e}\right)^{2}} \frac{\left|V_{u d} V_{u s}^{*}\right|^{2}}{\left[R_{A}^{2}+\left(1+\left|V_{u d}\right|^{2}\right)^{2}\right]},
$$

where

$$
R_{A} \equiv \frac{A_{S_{1}}}{A_{S_{2}}}=\left[\frac{\alpha_{1}\left(\mu_{\mathrm{SUSY}}\right)}{\alpha_{1}\left(\mu_{\mathrm{GUT}}\right)}\right]^{\frac{2}{33}}\left[\frac{\alpha_{1}\left(m_{Z}\right)}{\alpha_{1}\left(\mu_{\mathrm{SUSY}}\right)}\right]^{\frac{6}{41}}
$$

We find $R_{A} \simeq 1$ in a typical supersymmetric mass spectrum, and for $R_{A}=1$ we have: ${ }^{10}$

$$
\frac{\Gamma\left(p \rightarrow \pi^{0} \mu^{+}\right)_{\text {unflipped }}}{\Gamma\left(p \rightarrow \pi^{0} e^{+}\right)_{\text {unflipped }}} \simeq 0.008 .
$$

Hence, the branching fraction of the muon mode is predicted to be smaller than that of the electron mode by approximately two orders of magnitude in the unflipped SU(5) GUT. This prediction is again rather robust: the uncertainty is $\mathcal{O}(10) \%$, which mainly comes from the errors in the hadronic matrix elements. We note also that the contribution of the color-triplet Higgs exchange to these decay modes in supersymmetric SU(5) is suppressed by small Yukawa couplings, and thus is negligible unless there is flavor violation in the sfermion mass matrices [19].

To determine the predicted value of the ratio in flipped $\mathrm{SU}(5)$ given by eq. (5.1), we perform a parameter scan similar to that in refs. [51, 52]. We first write the Yukawa matrix $\lambda_{6}$ in the form

$$
\lambda_{6}=r_{6} M_{6},
$$

where $r_{6}$ is a real constant, which plays a role of a scale factor, and $M_{6}$ is a generic complex $3 \times 3$ matrix. We then scan $r_{6}$ with a logarithmic distribution over the range $\left(10^{-4}, 1\right)$ choosing a total of 1000 values. For each value of $r_{6}$, we generate 1000 random complex $3 \times 3$ matrices $M_{6}$ with each component taking a value of $\mathcal{O}(1)$.

${ }^{10}$ This result is consistent with the formula given in ref. [100]

$$
\left(\frac{\Gamma\left(p \rightarrow \mu^{+}+X\right)}{\Gamma\left(p \rightarrow e^{+}+X\right)}\right)_{X \text { nonstrange }}=\frac{\sin ^{2} \theta_{c} \cos ^{2} \theta_{c}}{\left(1+\cos ^{2} \theta_{c}\right)^{2}+1} \simeq 0.01
$$

where $\theta_{c}$ is the Cabibbo angle: $\sin \theta_{c} \simeq 0.2245$. 


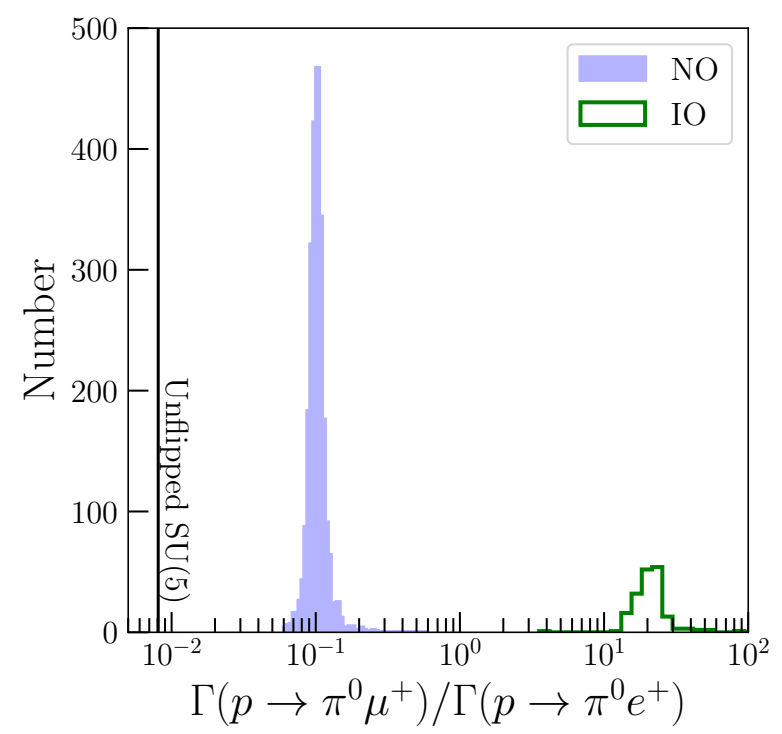

Figure 1. Histograms of $\Gamma\left(p \rightarrow \pi^{0} \mu^{+}\right) / \Gamma\left(p \rightarrow \pi^{0} e^{+}\right)$in the flipped SU(5) GUT model for the NO and IO cases in blue and green, respectively. The vertical line corresponds to the unflipped SU(5) prediction.

As discussed in refs. [51, 52], for each $3 \times 3$ matrix $\lambda_{6}$, the eigenvalues of the $m_{\nu}$ and $m_{\nu^{c}}$ matrices and the mixing matrices $U_{\nu^{c}}$ and $U_{\nu}$ are obtained as functions of $\mu^{1}$ and $\mu^{2}$ in eq. (2.5). We then determine these two $\mu$ parameters by requiring that the observed values of the squared mass differences, $\Delta m_{21}^{2} \equiv m_{2}^{2}-m_{1}^{2}$ and $\Delta m_{3 \ell}^{2} \equiv m_{3}^{2}-m_{\ell}^{2}$, are reproduced within the experimental uncertainties, where $\ell=1$ for the NO case and $\ell=2$ for the IO case. For the experimental input, we use the results from $\nu$-fit 4.0 given in refs. $[101,102]$. By using $U_{\nu}$ determined in this manner, we then compute the matrix $U_{l}$ using the relation (2.11). We parametrise the PMNS matrix elements following the RPP convention [83]:

$$
U_{\mathrm{PMNS}}=\left(\begin{array}{ccc}
c_{12} c_{13} & s_{12} c_{13} & s_{13} e^{-i \delta} \\
-s_{12} c_{23}-c_{12} s_{23} s_{13} e^{i \delta} & c_{12} c_{23}-s_{12} s_{23} s_{13} e^{i \delta} & s_{23} c_{13} \\
s_{12} s_{23}-c_{12} c_{23} s_{13} e^{i \delta} & -c_{12} s_{23}-s_{12} c_{23} s_{13} e^{i \delta} & c_{23} c_{13}
\end{array}\right)\left(\begin{array}{ccc}
1 & 0 & 0 \\
0 & e^{i \frac{\alpha_{2}}{2}} & 0 \\
0 & 0 & e^{i \frac{\alpha_{3}}{2}}
\end{array}\right),
$$

where $c_{i j} \equiv \cos \theta_{i j}$ and $s_{i j} \equiv \sin \theta_{i j}$ with the mixing angles $\theta_{i j}=[0, \pi / 2]$, the Dirac CP phase $\delta \in[0,2 \pi]$, and the order $m_{1}<m_{2}$ is chosen without loss of generality. Again we use the values obtained in refs. $[101,102]$ for $\theta_{12}, \theta_{23}, \theta_{13}$, and $\delta$. As for the Majorana phases $\alpha_{2}$ and $\alpha_{3}$, we set $\alpha_{2}=\alpha_{3}=0$ in this analysis since, as we shall see below, the result scarcely depends on these phases. We generate the same number of $\lambda_{6}$ matrices for each mass ordering, and find solutions for 2399 and 180 matrix choices for the NO and IO cases, respectively, out of a total of $10^{6}$ parameter sets sampled. This difference indicates some preference for the NO case in our model.

In figure 1 we display histograms of the ratio $\Gamma\left(p \rightarrow \pi^{0} \mu^{+}\right) / \Gamma\left(p \rightarrow \pi^{0} e^{+}\right)$in the NO and IO scenarios in blue and green, respectively. The vertical black solid line represents the predicted value in unflipped SU(5). As we see, the flipped SU(5) Model predicts this ratio 
to be $\sim 0.10$ and $\sim 23$ for the NO and IO cases, respectively. To understand the origin of these values, we first note that, due to the hierarchical structure of $m_{\nu}$ in eq. (2.9), $U_{\nu}$ has a simple form:

$$
U_{\nu} \simeq\left(\begin{array}{ccc}
1 & 0 & 0 \\
0 & \cos \theta & -\sin \theta \\
0 & \sin \theta & \cos \theta
\end{array}\right)
$$

for $\mathrm{NO}$, where $\sin \theta$ is found to be $\sim 0.38$, and

$$
U_{\nu} \simeq\left(\begin{array}{lll}
0 & 1 & 0 \\
0 & 0 & 1 \\
1 & 0 & 0
\end{array}\right)\left(\begin{array}{ccc}
1 & 0 & 0 \\
0 & 1 / \sqrt{2} & -1 / \sqrt{2} \\
0 & 1 / \sqrt{2} & 1 / \sqrt{2}
\end{array}\right)
$$

for IO, where the first matrix in the right-hand side arranges the order of the neutrino mass eigenvalues in accordance with the RPP convention. The relevant matrix elements of $U_{l}=U_{\mathrm{PMNS}}^{*} U_{\nu}$ are then given by

$$
\begin{aligned}
& \left(U_{l}\right)_{11} \simeq\left\{\begin{array}{ll}
\left(U_{\mathrm{PMNS}}^{*}\right)_{11}=c_{12} c_{13} & \mathrm{NO} \\
\left(U_{\mathrm{PMNS}}^{*}\right)_{13}=s_{13} e^{i \delta-i \frac{\alpha_{3}}{2}} & \mathrm{IO}
\end{array},\right. \\
& \left(U_{l}\right)_{21} \simeq \begin{cases}\left(U_{\mathrm{PMNS}}^{*}\right)_{21}=-s_{12} c_{23}-c_{12} s_{23} s_{13} e^{-i \delta} & \mathrm{NO} \\
\left(U_{\mathrm{PMNS}}^{*}\right)_{23}=s_{23} c_{12} e^{-i \frac{\alpha_{3}}{2}} & \mathrm{IO}\end{cases}
\end{aligned}
$$

which leads to

$$
\frac{\Gamma\left(p \rightarrow \pi^{0} \mu^{+}\right)_{\text {flipped }}}{\Gamma\left(p \rightarrow \pi^{0} e^{+}\right)_{\text {flipped }}} \simeq \frac{\left(\left\langle\pi^{0}\left|(u d)_{R} u_{L}\right| p\right\rangle_{\mu}\right)^{2}}{\left(\left\langle\pi^{0}\left|(u d)_{R} u_{L}\right| p\right\rangle_{e}\right)^{2}} \frac{\left|s_{12} c_{23}+c_{12} s_{23} s_{13} e^{-i \delta}\right|^{2}}{\left(c_{12} c_{13}\right)^{2}} \simeq 0.10,
$$

for $\mathrm{NO}$, and

$$
\frac{\Gamma\left(p \rightarrow \pi^{0} \mu^{+}\right)_{\text {flipped }}}{\Gamma\left(p \rightarrow \pi^{0} e^{+}\right)_{\text {flipped }}} \simeq \frac{\left(\left\langle\pi^{0}\left|(u d)_{R} u_{L}\right| p\right\rangle_{\mu}\right)^{2}}{\left(\left\langle\pi^{0}\left|(u d)_{R} u_{L}\right| p\right\rangle_{e}\right)^{2}} \frac{\left(s_{23} c_{12}\right)^{2}}{s_{13}^{2}} \simeq 22.9,
$$

for IO. These approximate estimates are in good agreement with the results given in figure 1. We also note that these two expressions do not depend on the unknown Majorana phases, $\alpha_{2}$ and $\alpha_{3}$. As a consequence, although we have fixed these phases to be zero in our analysis, we expect that the results in figure 1 will not be changed even if we take different values for these phases.

The values of $\Gamma\left(p \rightarrow \pi^{0} \mu^{+}\right) / \Gamma\left(p \rightarrow \pi^{0} e^{+}\right)$predicted in the NO and IO flipped SU(5) scenarios are rather insensitive to the mass of the lightest neutrino, as seen in figure 2. On the other hand, we also see there that the spread in predicted values increases with the lightest neutrino mass. It may be challenging for the envisaged next-generation neutrino experiments to measure any deviation from the central values of the model predictions, but the NO and IO predictions remain well separated and hence distinguishable.

The predicted values of $\Gamma\left(p \rightarrow \pi^{0} \mu^{+}\right) / \Gamma\left(p \rightarrow \pi^{0} e^{+}\right)$in flipped SU(5) are much larger than the standard unflipped $\mathrm{SU}(5)$ prediction, which is $\simeq 0.008$. We may therefore be 


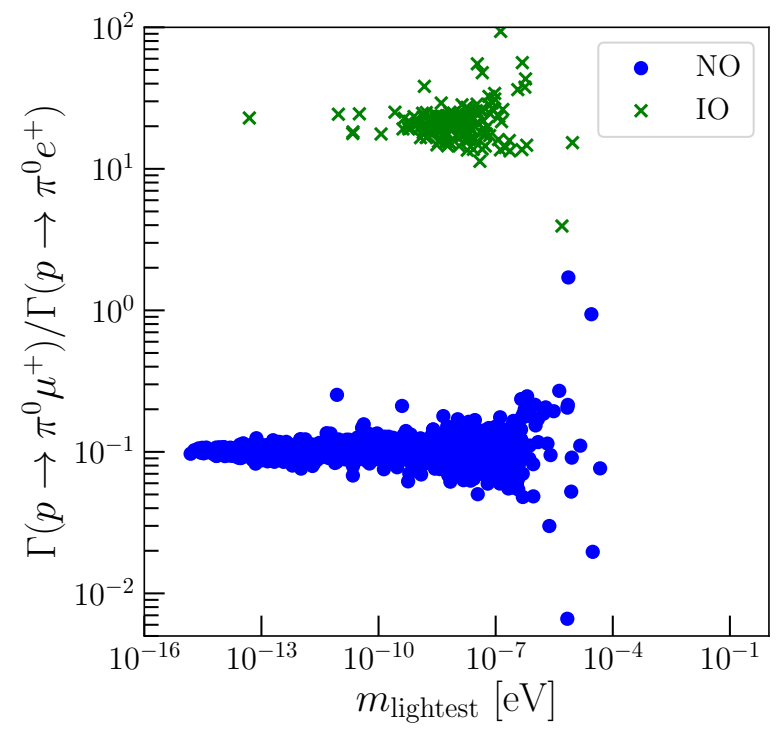

Figure 2. Scatter plots of values of $\Gamma\left(p \rightarrow \pi^{0} \mu^{+}\right) / \Gamma\left(p \rightarrow \pi^{0} e^{+}\right)$in the flipped SU(5) GUT model for the NO and IO cases (blue and green, respectively), as functions of the lightest neutrino mass.

able to distinguish these two models in future proton decay experiments by measuring the partial lifetimes of these two decay modes. We can also determine the neutrino mass ordering in the case of flipped SU(5). Proton decay experiments are relatively sensitive to both of these decay modes, leading to the strongest available constraints on proton partial lifetimes: the current limit on $\tau\left(p \rightarrow \pi^{0} e^{+}\right)$from Super-Kamiokande is $2.4 \times 10^{34} \mathrm{yrs}$ and that on $\tau\left(p \rightarrow \pi^{0} \mu^{+}\right)$is $1.6 \times 10^{34}$ yrs $[103,104]$ which can be compared to the predicted partial lifetimes given in eq. (3.12) and (3.15), respectively. This makes the ratio $\Gamma\left(p \rightarrow \pi^{0} \mu^{+}\right) / \Gamma\left(p \rightarrow \pi^{0} e^{+}\right)$given in eq. (5.1) interesting for testing the prediction of flipped $\mathrm{SU}(5)$ in future proton decay experiments such as Hyper-Kamiokande [5].

\section{$5.2 \sum_{i} \Gamma\left(p \rightarrow \pi^{+} \bar{\nu}_{i}\right) / \Gamma\left(p \rightarrow \pi^{0} e^{+}\right)$}

Next we consider the ratio $\sum_{i} \Gamma\left(p \rightarrow \pi^{+} \bar{\nu}_{i}\right) / \Gamma\left(p \rightarrow \pi^{0} e^{+}\right)$. Eqs. (3.21) and (3.11) imply that for the flipped $\mathrm{SU}(5)$ we have

$$
\frac{\sum_{i} \Gamma\left(p \rightarrow \pi^{+} \bar{\nu}_{i}\right)_{\text {flipped }}}{\Gamma\left(p \rightarrow \pi^{0} e^{+}\right)_{\text {flipped }}}=\frac{\left(\left\langle\pi^{+}\left|(u d)_{R} d_{L}\right| p\right\rangle\right)^{2}}{\left(\left\langle\pi^{0}\left|(u d)_{R} u_{L}\right| p\right\rangle_{e}\right)^{2}} \frac{1}{\left|V_{u d}\right|^{2}\left|\left(U_{l}\right)_{11}\right|^{2}},
$$

whereas for unflipped $\mathrm{SU}(5)$ we can use eqs. (4.4) and (4.2) to obtain

$$
\frac{\sum_{i} \Gamma\left(p \rightarrow \pi^{+} \bar{\nu}_{i}\right)_{\text {unflipped }}}{\Gamma\left(p \rightarrow \pi^{0} e^{+}\right)_{\text {unflipped }}}=\frac{\left(\left\langle\pi^{+}\left|(u d)_{R} d_{L}\right| p\right\rangle\right)^{2}}{\left(\left\langle\pi^{0}\left|(u d)_{R} u_{L}\right| p\right\rangle_{e}\right)^{2}} \frac{R_{A}^{2}\left|V_{u d}\right|^{2}}{\left[R_{A}^{2}+\left(1+\left|V_{u d}\right|^{2}\right)^{2}\right]} .
$$

Setting $R_{A}=1$ again, we find

$$
\frac{\sum_{i} \Gamma\left(p \rightarrow \pi^{+} \bar{\nu}_{i}\right)_{\text {unflipped }}}{\Gamma\left(p \rightarrow \pi^{0} e^{+}\right)_{\text {unflipped }}} \simeq 0.4
$$




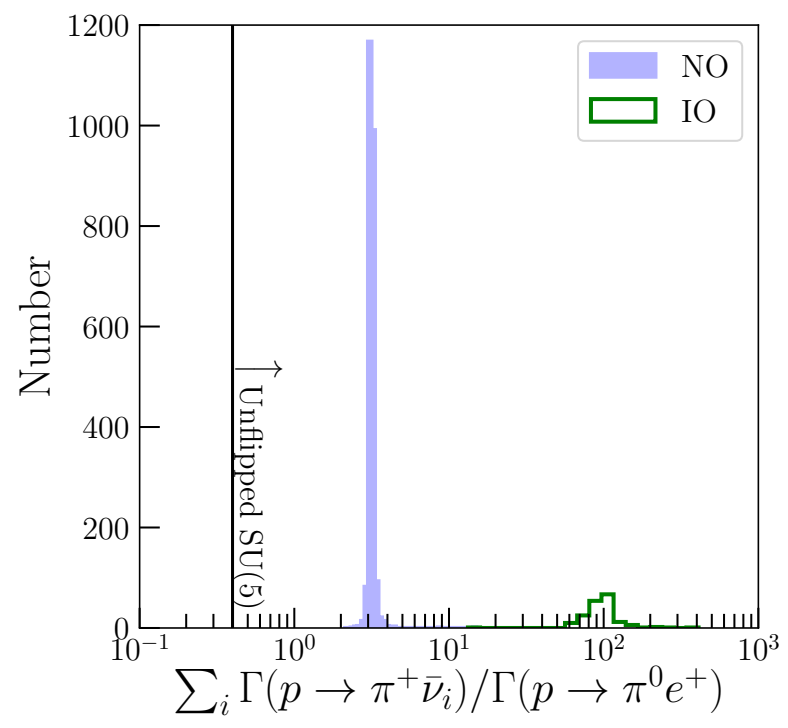

Figure 3. Histograms of $\sum_{i} \Gamma\left(p \rightarrow \pi^{+} \bar{\nu}_{i}\right) / \Gamma\left(p \rightarrow \pi^{0} e^{+}\right)$in flipped $\mathrm{SU}(5)$ for the NO and IO cases in blue and green, respectively. The unflipped $\mathrm{SU}(5)$ prediction has a lower limit shown as the vertical solid line.

We note, however, that in the supersymmetric standard SU(5) GUT colour-triplet Higgs exchange also induces $p \rightarrow \pi^{+} \bar{\nu}$ (see, for instance, refs. $[13,19,20,23]$ ), which can be much larger than the contribution in eq. (4.4). Therefore, the value in eq. (5.15) should be regarded as a lower limit on $\sum_{i} \Gamma\left(p \rightarrow \pi^{+} \bar{\nu}_{i}\right) / \Gamma\left(p \rightarrow \pi^{0} e^{+}\right)$in standard unflipped SU(5).

We show in figure 3 histograms of $\sum_{i} \Gamma\left(p \rightarrow \pi^{+} \bar{\nu}_{i}\right) / \Gamma\left(p \rightarrow \pi^{0} e^{+}\right)$in the flipped SU(5) model for the NO and IO cases in blue and green, respectively. Unflipped SU(5) has the lower limit indicated by the vertical solid line. As in the previous subsection, we can again estimate this ratio using the approximation given in eq. (5.9):

$$
\frac{\sum_{i} \Gamma\left(p \rightarrow \pi^{+} \bar{\nu}_{i}\right)_{\text {flipped }}}{\Gamma\left(p \rightarrow \pi^{0} e^{+}\right)_{\text {flipped }}}=\frac{\left(\left\langle\pi^{+}\left|(u d)_{R} d_{L}\right| p\right\rangle\right)^{2}}{\left(\left\langle\pi^{0}\left|(u d)_{R} u_{L}\right| p\right\rangle_{e}\right)^{2}} \frac{1}{\left|V_{u d}\right|^{2}\left(c_{12} c_{13}\right)^{2}} \simeq 3.15,
$$

for $\mathrm{NO}$, and

$$
\frac{\sum_{i} \Gamma\left(p \rightarrow \pi^{+} \bar{\nu}_{i}\right)_{\text {flipped }}}{\Gamma\left(p \rightarrow \pi^{0} e^{+}\right)_{\text {flipped }}}=\frac{\left(\left\langle\pi^{+}\left|(u d)_{R} d_{L}\right| p\right\rangle\right)^{2}}{\left(\left\langle\pi^{0}\left|(u d)_{R} u_{L}\right| p\right\rangle_{e}\right)^{2}} \frac{1}{\left|V_{u d}\right|^{2} s_{13}^{2}} \simeq 94.8,
$$

for IO, which agree with the results shown in figure 3.

This ratio is, however, less powerful for distinguishing the flipped and unflipped SU(5) GUTs than $\Gamma\left(p \rightarrow \pi^{0} \mu^{+}\right) / \Gamma\left(p \rightarrow \pi^{0} e^{+}\right)$. First, due to the potential contribution of the colour-triplet Higgs exchange, we have only a lower limit on the unflipped SU(5) prediction. Since the predicted values in the flipped $\mathrm{SU}(5)$ are larger than this lower limit, the unflipped SU(5) prediction can in principle mimic the flipped SU(5) predictions. Secondly, the sensitivities of experiments to $p \rightarrow \pi^{+} \bar{\nu}$ and $n \rightarrow \pi^{0} \bar{\nu}$ tend to be much worse than that to $p \rightarrow \pi^{0} \mu^{+}$; the present bound on $p \rightarrow \pi^{+} \bar{\nu}$ from SuperKamiokande is $\tau\left(p \rightarrow \pi^{+} \bar{\nu}\right)>3.9 \times 10^{32}$ yrs and that on $\tau\left(n \rightarrow \pi^{0} \bar{\nu}\right)>1.1 \times 10^{33}$ yrs [105], 
which are much lower than the limit on $p \rightarrow \pi^{0} \mu^{+}$. On the other hand, the value of $\sum_{i} \Gamma\left(p \rightarrow \pi^{+} \bar{\nu}_{i}\right) / \Gamma\left(p \rightarrow \pi^{0} e^{+}\right)$predicted in the flipped SU(5) model in the IO case is so large that this might be detectable.

\section{$5.3 \quad \Gamma\left(p \rightarrow K^{0} e^{+}\right) / \Gamma\left(p \rightarrow \pi^{0} e^{+}\right)$}

The ratio $\Gamma\left(p \rightarrow K^{0} e^{+}\right) / \Gamma\left(p \rightarrow \pi^{0} e^{+}\right)$in flipped $\mathrm{SU}(5)$ is computed from eqs. (3.27) and (3.11) to be

$$
\frac{\Gamma\left(p \rightarrow K^{0} e^{+}\right)_{\text {flipped }}}{\Gamma\left(p \rightarrow \pi^{0} e^{+}\right)_{\text {flipped }}}=\frac{\left(m_{p}^{2}-m_{K}^{2}\right)^{2}}{\left(m_{p}^{2}-m_{\pi}^{2}\right)^{2}} \frac{\left(\left\langle K^{0}\left|(u s)_{R} u_{L}\right| p\right\rangle_{e}\right)^{2}}{\left(\left\langle\pi^{0}\left|(u d)_{R} u_{L}\right| p\right\rangle_{e}\right)^{2}} \frac{\left|V_{u s}\right|^{2}}{\left|V_{u d}\right|^{2}} \simeq 1.8 \times 10^{-2} .
$$

As we see, this ratio does not depend on the matrix $U_{l}$. In unflipped $\mathrm{SU}(5)$, we use eqs. (4.5) and (4.2) to find

$$
\frac{\Gamma\left(p \rightarrow K^{0} e^{+}\right)_{\text {unflipped }}}{\Gamma\left(p \rightarrow \pi^{0} e^{+}\right)_{\text {unflipped }}}=\frac{\left(m_{p}^{2}-m_{K}^{2}\right)^{2}}{\left(m_{p}^{2}-m_{\pi}^{2}\right)^{2}} \frac{\left(\left\langle K^{0}\left|(u s)_{R} u_{L}\right| p\right\rangle_{e}\right)^{2}}{\left(\left\langle\pi^{0}\left|(u d)_{R} u_{L}\right| p\right\rangle_{e}\right)^{2}} \frac{\left|V_{u d} V_{u s}^{*}\right|^{2}}{\left[R_{A}^{2}+\left(1+\left|V_{u d}\right|^{2}\right)^{2}\right]} \simeq 3.3 \times 10^{-3},
$$

for $R_{A}=1$. The contribution of the colour-triplet Higgs exchange to $p \rightarrow K^{0} e^{+}$is negligible unless flavour violation occurs in sfermion mass matrices $[19,20]$, so this value can be regarded as a prediction of unflipped $\mathrm{SU}(5)$. As we see, this unflipped $\mathrm{SU}(5)$ prediction is much lower than the flipped SU(5) prediction (5.18), and thus we can in principle also use the ratio $\Gamma\left(p \rightarrow K^{0} e^{+}\right) / \Gamma\left(p \rightarrow \pi^{0} e^{+}\right)$to distinguish between these two GUT models.

\section{$5.4 \Gamma\left(p \rightarrow K^{0} \mu^{+}\right) / \Gamma\left(p \rightarrow \pi^{0} \mu^{+}\right)$}

From eqs. (3.28) and (3.14), we have

$$
\frac{\Gamma\left(p \rightarrow K^{0} \mu^{+}\right)_{\text {flipped }}}{\Gamma\left(p \rightarrow \pi^{0} \mu^{+}\right)_{\text {flipped }}}=\frac{\left(m_{p}^{2}-m_{K}^{2}\right)^{2}}{\left(m_{p}^{2}-m_{\pi}^{2}\right)^{2}} \frac{\left(\left\langle K^{0}\left|(u s)_{R} u_{L}\right| p\right\rangle_{\mu}\right)^{2}}{\left(\left\langle\pi^{0}\left|(u d)_{R} u_{L}\right| p\right\rangle_{\mu}\right)^{2}} \frac{\left|V_{u s}\right|^{2}}{\left|V_{u d}\right|^{2}} \simeq 0.02 .
$$

Again, this ratio does not depend on the matrix $U_{l}$. In unflipped SU(5), eqs. (4.6) and (4.3) lead to

$$
\frac{\Gamma\left(p \rightarrow K^{0} \mu^{+}\right)_{\text {unflipped }}}{\Gamma\left(p \rightarrow \pi^{0} \mu^{+}\right)_{\text {unflipped }}}=\frac{\left(m_{p}^{2}-m_{K}^{2}\right)^{2}}{\left(m_{p}^{2}-m_{\pi}^{2}\right)^{2}} \frac{\left(\left\langle K^{0}\left|(u s)_{R} u_{L}\right| p\right\rangle_{\mu}\right)^{2}}{\left(\left\langle\pi^{0}\left|(u d)_{R} u_{L}\right| p\right\rangle_{\mu}\right)^{2}} \frac{\left[R_{A}^{2}+\left(1+\left|V_{u s}\right|^{2}\right)^{2}\right]}{\left|V_{u d} V_{u s}^{*}\right|^{2}} \simeq 16.7
$$

for $R_{A}=1$. The contribution of colour-triplet Higgs exchange to $p \rightarrow K^{0} \mu^{+}$is small unless flavour violation occurs in sfermion mass matrices $[19,20]$. Therefore, this ratio can again be used to distinguish between the flipped and unflipped SU(5) GUTs.

\section{$5.5 p \rightarrow K^{+} \bar{\nu}$}

This process tends to be the dominant decay mode in the supersymmetric standard unflipped SU(5) GUT model [11, 12]. In flipped SU(5), on the other hand, as seen in eq. (3.32), we have [68]

$$
\Gamma\left(p \rightarrow K^{+} \bar{\nu}_{i}\right)=0
$$




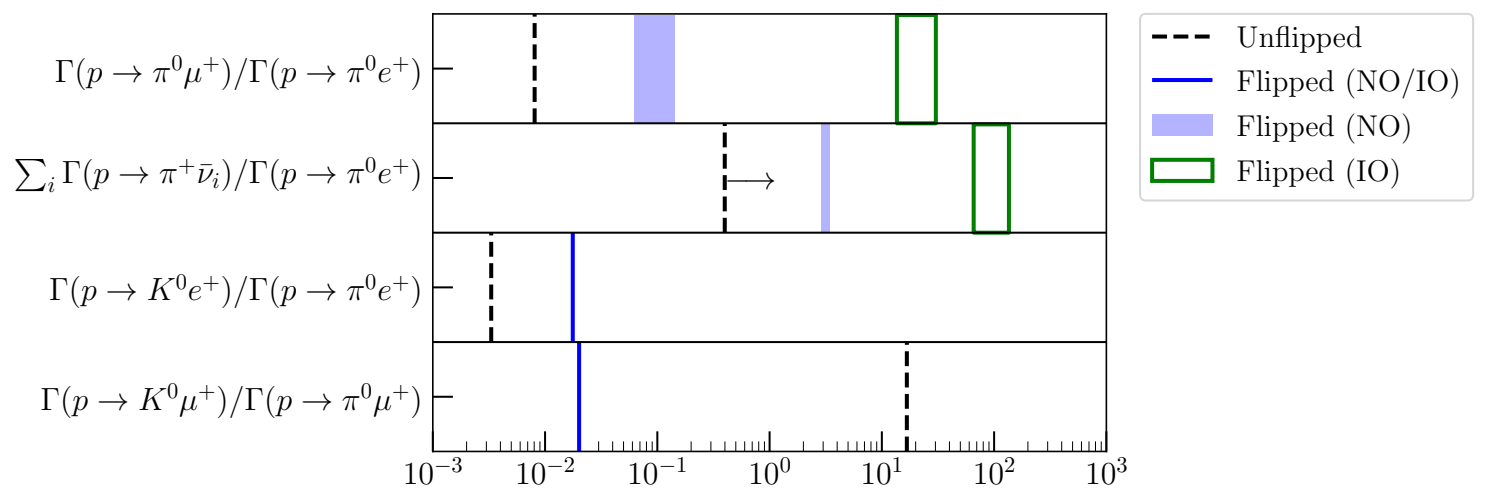

Figure 4. Compilation of the ratios of proton decay rates predicted in standard unflipped SU(5) (dashed black lines), no-scale flipped SU(5) with neutrino masses that are normal-ordered (NO, blue shading) or inverse-ordered (IO, green boxes). Cases where the flipped $\mathrm{SU}(5)$ predictions are independent of the neutrino mass ordering are indicated by solid blue lines.

This is a distinctive prediction in flipped $\mathrm{SU}(5)$ - if this decay mode is discovered in future proton decay experiments, flipped $\mathrm{SU}(5)$ is excluded.

\section{Discussion and prospects}

We have explored in this paper various nucleon decay modes in the flipped SU(5) GUT model developed in [49-52], which builds upon earlier studies [37-43, 68]. We have presented flipped $\mathrm{SU}(5)$ predictions in scenarios with both normal-ordered neutrino masses (NO) and inverse ordering (IO), and compared them with the predictions of the standard unflipped SU(5) GUT. Our results for the ratios of decay rates $\Gamma\left(p \rightarrow \pi^{0} \mu^{+}\right) / \Gamma\left(p \rightarrow \pi^{0} e^{+}\right)$, $\Gamma\left(p \rightarrow \pi^{+} \bar{\nu}\right) / \Gamma\left(p \rightarrow \pi^{0} e^{+}\right), \Gamma\left(p \rightarrow K^{0} e^{+}\right) / \Gamma\left(p \rightarrow \pi^{0} e^{+}\right)$and $\Gamma\left(p \rightarrow K^{0} \mu^{+}\right) / \Gamma\left(p \rightarrow \pi^{0} \mu^{+}\right)$ are compiled in figure $4 .{ }^{11}$ In all cases we see clear differences between the predictions of flipped SU(5) and standard SU(5), and in the cases of $\Gamma\left(p \rightarrow \pi^{0} \mu^{+}\right) / \Gamma\left(p \rightarrow \pi^{0} e^{+}\right)$ and $\Gamma\left(p \rightarrow \pi^{+} \bar{\nu}\right) / \Gamma\left(p \rightarrow \pi^{0} e^{+}\right)$we also see clear distinctions between the NO and IO predictions.

The 'Golden Ratio' from the point of view of our analysis is $\Gamma\left(p \rightarrow \pi^{0} \mu^{+}\right) / \Gamma(p \rightarrow$ $\left.\pi^{0} e^{+}\right)$. We recall that Super-Kamiokande has similar sensitivities to these two decay modes, and has established limits on their partial lifetimes of 1.6 and $2.4 \times 10^{34}$ yrs, respectively $[103,104] .{ }^{12}$ We expect that future proton decay experiments such as HyperKamiokande [5] should have an order of magnitude greater sensitivity to both these decay modes, and hence have a window of opportunity to probe both the NO and IO predictions. Indeed, in the IO case a search for $p \rightarrow \pi^{0} \mu^{+}$with a sensitivity to a partial lifetime of $10^{35}$ yrs would constrain the model as much as a sensitivity to $p \rightarrow \pi^{0} e^{+}$of about $2 \times 10^{36}$ yrs.

\footnotetext{
${ }^{11}$ We note also the flipped $\mathrm{SU}(5)$ prediction that $\Gamma\left(p \rightarrow K^{+} \bar{\nu}\right)$ vanishes.

${ }^{12}$ The corresponding searches for $n \rightarrow \pi^{-} l^{+}$are less constraining; the present limits on the lifetimes of these decay modes, $\tau\left(n \rightarrow \pi^{-} e^{+}\right)>5.3 \times 10^{33}$ yrs and $\tau\left(n \rightarrow \pi^{-} \mu^{+}\right)>3.5 \times 10^{33}$ yrs [106], are weaker than those on $p \rightarrow \pi^{0} l^{+}$, while the predicted partial decay widths are larger, as shown in eq. (3.16).
} 
Our results highlight the importance of targeting proton decay modes involving finalstate particles from different generations, since our 'Golden Ratio' and two others, $\Gamma(p \rightarrow$ $\left.K^{0} e^{+}\right) / \Gamma\left(p \rightarrow \pi^{0} e^{+}\right)$and $\Gamma\left(p \rightarrow K^{0} \mu^{+}\right) / \Gamma\left(p \rightarrow \pi^{0} \mu^{+}\right)$, involve mixtures of first- and second-generation leptons and quarks.

The fourth ratio, $\Gamma\left(p \rightarrow \pi^{+} \bar{\nu}\right) / \Gamma\left(p \rightarrow \pi^{0} e^{+}\right)$, does not involve identifiable secondgeneration fermions, but second- and third-generation neutrinos contribute to the enhanced values of the ratio predicted in the two flipped $\mathrm{SU}(5)$ scenarios we have studied. The current limit on $\tau\left(p \rightarrow \pi^{+} \bar{\nu}\right)$ is only $3.9 \times 10^{32}$ yrs [105]. However, in the IO model this lifetime would be two orders of magnitude shorter than $\tau\left(p \rightarrow \pi^{0} e^{+}\right)$, so the current limit corresponds to $\tau\left(p \rightarrow \pi^{0} e^{+}\right)>3.7 \times 10^{34}$ yrs. Hence the search for $p \rightarrow \pi^{+} \bar{\nu}$ currently sets a tighter constraint on the IO model than that set by the $p \rightarrow \pi^{0} e^{+}$search. We are unaware of estimates of the improved sensitivity to $p \rightarrow \pi^{+} \bar{\nu}$ of the upcoming large neutrino experiments, but increasing the sensitivity to $p \rightarrow \pi^{+} \bar{\nu}$ by the same factor as anticipated for $p \rightarrow \pi^{0} e^{+}[5]$ would constrain the IO model as much as a sensitivity to the latter mode of $>3 \times 10^{35} \mathrm{yrs}$. The current limit $\tau\left(n \rightarrow \pi^{0} \bar{\nu}\right)>1.1 \times 10^{33} \mathrm{yrs}[105]$ constrains the IO model even more, since it corresponds to $\tau\left(p \rightarrow \pi^{0} e^{+}\right)>5 \times 10^{34}$ yrs. Again, we are unaware of any estimate of the sensitivity in a future experiment, but an order-of-magnitude improvement would correspond to $\tau\left(p \rightarrow \pi^{0} e^{+}\right)>5 \times 10^{35}$ yrs.

We have focused in this paper on the contributions of dimension- 6 operators to nucleon decay. This is sufficient for the flipped $\mathrm{SU}(5)$ case, since dimension-5 proton decay is suppressed due to the absence of a vector-like mass term for the $H$ and $\bar{H}$ fields. For unflipped SU(5), on the other hand, dimension-5 operators can in general give a significant contribution to nucleon decay, though these may suppressed in the presence of a discrete symmetry such as hexality [36] or a missing-partner mechanism. If present, dimension-5 operators may induce $p \rightarrow K^{+} \bar{\nu}$ with a sizable rate $[9,10]$. In fact, it was found in the constrained minimal supersymmetric Standard Model that the partial lifetime of $p \rightarrow K^{+} \bar{\nu}$ could well be within the reach of future proton decay experiments [13]. If this decay mode is indeed discovered in the future, then the flipped SU(5) GUT model is excluded. The dimension- 5 operators also induce $p \rightarrow \pi^{+} \bar{\nu}$, whereas their contribution to the decay modes with a charged lepton is negligible unless there is flavor violation in sfermion masses [19]. As a result, the ratio $\Gamma\left(p \rightarrow \pi^{+} \bar{\nu}\right) / \Gamma\left(p \rightarrow \pi^{0} e^{+}\right)$is predicted to be larger, as shown in the arrow in figure 4 , while the other ratios remain unchanged in the presence of the dimension5 operators. In particular, the 'Golden Ratio', $\Gamma\left(p \rightarrow \pi^{0} \mu^{+}\right) / \Gamma\left(p \rightarrow \pi^{0} e^{+}\right)$, is still useful to discriminate the flipped and unflipped GUT models.

These examples show that if the upcoming large neutrino experiments do discover nucleon decay, they will have interesting opportunities to explore both GUT and flavour physics.

\section{Acknowledgments}

The work of J.E. was supported partly by the United Kingdom STFC Grant ST/P000258/1 and partly by the Estonian Research Council via a Mobilitas Pluss grant. The work of M.A.G.G. was supported by the Spanish Agencia Estatal de Investigación through the 
grants FPA2015-65929-P (MINECO/FEDER, UE), PGC2018095161-B-I00, IFT Centro de Excelencia Severo Ochoa SEV-2016-0597, and Red Consolider MultiDark FPA201790566-REDC. The work of N.N. was supported by the Grant-in-Aid for Young Scientists B (No. 17K14270) and Innovative Areas (No.18H05542). N.N. would also like to thank the members of William I. Fine Theoretical Physics Institute for their hospitality and financial support while finishing this work. The work of D.V.N. was supported partly by the DOE grant DE-FG02-13ER42020 and partly by the Alexander S. Onassis Public Benefit Foundation. The work of K.A.O. was supported partly by the DOE grant DE-SC0011842 at the University of Minnesota.

Open Access. This article is distributed under the terms of the Creative Commons Attribution License (CC-BY 4.0), which permits any use, distribution and reproduction in any medium, provided the original author(s) and source are credited.

\section{References}

[1] JUNO collaboration, Neutrino Physics with JUNO, J. Phys. G 43 (2016) 030401 [arXiv: 1507.05613] [INSPIRE].

[2] DUNE collaboration, Long-Baseline Neutrino Facility (LBNF) and Deep Underground Neutrino Experiment (DUNE): Conceptual Design Report Volume 2: The Physics Program for DUNE at LBNF, arXiv: 1512.06148 [INSPIRE].

[3] DUNE collaboration, The DUNE Far Detector Interim Design Report Volume 1: Physics, Technology and Strategies, arXiv:1807.10334 [INSPIRE].

[4] DUNE collaboration, Deep Underground Neutrino Experiment (DUNE), Far Detector Technical Design Report, Volume II DUNE Physics, arXiv:2002.03005 [INSPIRE].

[5] Hyper-Kamiokande collaboration, Hyper-Kamiokande Design Report, arXiv: 1805.04163 [INSPIRE].

[6] H. Georgi and S.L. Glashow, Unity of All Elementary Particle Forces, Phys. Rev. Lett. 32 (1974) 438 [INSPIRE].

[7] S. Dimopoulos and H. Georgi, Softly Broken Supersymmetry and SU(5), Nucl. Phys. B 193 (1981) 150 [INSPIRE].

[8] N. Sakai, Naturalness in Supersymmetric Guts, Z. Phys. C 11 (1981) 153 [InSPIRE].

[9] S. Dimopoulos, S. Raby and F. Wilczek, Proton Decay in Supersymmetric Models, Phys. Lett. 112B (1982) 133 [INSPIRE].

[10] J.R. Ellis, D.V. Nanopoulos and S. Rudaz, GUTs 3: SUSY GUTs 2, Nucl. Phys. B 202 (1982) 43 [INSPIRE].

[11] N. Sakai and T. Yanagida, Proton Decay in a Class of Supersymmetric Grand Unified Models, Nucl. Phys. B 197 (1982) 533 [InSPIRE].

[12] S. Weinberg, Supersymmetry at Ordinary Energies. 1. Masses and Conservation Laws, Phys. Rev. D 26 (1982) 287 [InSPIRE].

[13] J. Ellis, J.L. Evans, N. Nagata, K.A. Olive and L. Velasco-Sevilla, Supersymmetric Proton Decay Revisited, Eur. Phys. J. C 80 (2020) 332 [arXiv:1912.04888] [INSPIRE]. 
[14] S. Weinberg, Baryon and Lepton Nonconserving Processes, Phys. Rev. Lett. 43 (1979) 1566 [INSPIRE].

[15] F. Wilczek and A. Zee, Operator Analysis of Nucleon Decay, Phys. Rev. Lett. 43 (1979) 1571 [INSPIRE].

[16] T. Goto and T. Nihei, Effect of RRRR dimension five operator on the proton decay in the minimal SU(5) SUGRA GUT model, Phys. Rev. D 59 (1999) 115009 [hep-ph/9808255] [INSPIRE].

[17] H. Murayama and A. Pierce, Not even decoupling can save minimal supersymmetric SU(5), Phys. Rev. D 65 (2002) 055009 [hep-ph/0108104] [INSPIRE].

[18] J. Hisano, D. Kobayashi, T. Kuwahara and N. Nagata, Decoupling Can Revive Minimal Supersymmetric SU(5), JHEP 07 (2013) 038 [arXiv: 1304.3651] [INSPIRE].

[19] N. Nagata and S. Shirai, Sfermion Flavor and Proton Decay in High-Scale Supersymmetry, JHEP 03 (2014) 049 [arXiv: 1312.7854] [INSPIRE].

[20] N. Nagata, Proton Decay in High-scale Supersymmetry, Ph.D. Thesis (2014) [DOI].

[21] J.L. Evans, N. Nagata and K.A. Olive, SU(5) Grand Unification in Pure Gravity Mediation, Phys. Rev. D 91 (2015) 055027 [arXiv: 1502.00034] [inSPIRE].

[22] J. Ellis, J.L. Evans, F. Luo, N. Nagata, K.A. Olive and P. Sandick, Beyond the CMSSM without an Accelerator: Proton Decay and Direct Dark Matter Detection, Eur. Phys. J. C 76 (2016) 8 [arXiv: 1509.08838] [INSPIRE].

[23] J. Ellis, J.L. Evans, A. Mustafayev, N. Nagata and K.A. Olive, The Super-GUT CMSSM Revisited, Eur. Phys. J. C 76 (2016) 592 [arXiv: 1608.05370] [InSPIRE].

[24] J.L. Evans, N. Nagata and K.A. Olive, A Minimal SU(5) SuperGUT in Pure Gravity Mediation, Eur. Phys. J. C 79 (2019) 490 [arXiv: 1902.09084] [INSPIRE].

[25] D. McKeen, M. Pospelov and A. Ritz, Electric dipole moment signatures of PeV-scale superpartners, Phys. Rev. D 87 (2013) 113002 [arXiv:1303.1172] [INSPIRE].

[26] M. Liu and P. Nath, Higgs boson mass, proton decay, naturalness and constraints of the LHC and Planck data, Phys. Rev. D 87 (2013) 095012 [arXiv: 1303.7472] [InSPIRE].

[27] B. Bajc, S. Lavignac and T. Mede, Resurrecting the minimal renormalizable supersymmetric SU(5) model, JHEP 01 (2016) 044 [arXiv: 1509.06680] [INSPIRE].

[28] J.L. Evans, K. Kadota and T. Kuwahara, Revisiting Flavor and CP-violation in Supersymmetric SU(5) with Right-Handed Neutrinos, Phys. Rev. D 98 (2018) 075030 [arXiv: 1807.08234] [INSPIRE].

[29] S. Pokorski, K. Rolbiecki, G.G. Ross and K. Sakurai, A new approach to gauge coupling unification and proton decay, JHEP 04 (2019) 161 [arXiv:1902.06093] [INSPIRE].

[30] ATLAS collaboration, Search for supersymmetry in final states with missing transverse momentum and multiple b-jets in proton-proton collisions at $\sqrt{s}=13$ TeV with the ATLAS detector, JHEP 06 (2018) 107 [arXiv:1711.01901] [INSPIRE].

[31] ATLAS collaboration, Search for squarks and gluinos in final states with jets and missing transverse momentum using $36 \mathrm{fb}^{-1}$ of $\sqrt{\mathrm{s}}=13 \mathrm{TeV}$ pp collision data with the ATLAS detector, Phys. Rev. D 97 (2018) 112001 [arXiv:1712.02332] [INSPIRE].

[32] CMS collaboration, Search for new phenomena with the $M_{\mathrm{T} 2}$ variable in the all-hadronic final state produced in proton-proton collisions at $\sqrt{s}=13$ TeV, Eur. Phys. J. C 77 (2017) 710 [arXiv: 1705.04650] [INSPIRE]. 
[33] CMS collaboration, Search for natural and split supersymmetry in proton-proton collisions at $\sqrt{s}=13 \mathrm{TeV}$ in final states with jets and missing transverse momentum, JHEP 05 (2018) 025 [arXiv: 1802.02110] [INSPIRE].

[34] ATLAS collaboration, https://twiki.cern.ch/twiki/bin/view/AtlasPublic/SupersymmetryPublicResults.

[35] CMS collaboration, https://twiki.cern.ch/twiki/bin/view/CMSPublic/PhysicsResultsSUS.

[36] S. Förste, H.P. Nilles, S. Ramos-Sanchez and P.K.S. Vaudrevange, Proton Hexality in Local Grand Unification, Phys. Lett. B 693 (2010) 386 [arXiv:1007.3915] [InSPIRE].

[37] S.M. Barr, A New Symmetry Breaking Pattern for $\mathrm{SO}(10)$ and Proton Decay, Phys. Lett. 112B (1982) 219 [INSPIRE].

[38] S.M. Barr, Some Comments on Flipped $\mathrm{SU}(5) \times \mathrm{U}(1)$ and Flipped Unification in General, Phys. Rev. D 40 (1989) 2457 [InSPIRE].

[39] J.P. Derendinger, J.E. Kim and D.V. Nanopoulos, Anti-SU(5), Phys. Lett. 139B (1984) 170 [INSPIRE].

[40] I. Antoniadis, J.R. Ellis, J.S. Hagelin and D.V. Nanopoulos, Supersymmetric Flipped SU(5) Revitalized, Phys. Lett. B 194 (1987) 231 [INSPIRE].

[41] I. Antoniadis, J.R. Ellis, J.S. Hagelin and D.V. Nanopoulos, GUT Model Building with Fermionic Four-Dimensional Strings, Phys. Lett. B 205 (1988) 459 [inSPIRE].

[42] I. Antoniadis, J.R. Ellis, J.S. Hagelin and D.V. Nanopoulos, An Improved SU(5) $\times$ U(1) Model from Four-Dimensional String, Phys. Lett. B 208 (1988) 209 [InSPIRE].

[43] I. Antoniadis, J.R. Ellis, J.S. Hagelin and D.V. Nanopoulos, The Flipped SU(5) $\times$ U(1) String Model Revamped, Phys. Lett. B 231 (1989) 65 [InSPIRE].

[44] A. Masiero, D.V. Nanopoulos, K. Tamvakis and T. Yanagida, Naturally Massless Higgs Doublets in Supersymmetric SU(5), Phys. Lett. 115B (1982) 380 [INSPIRE].

[45] B. Grinstein, A Supersymmetric SU(5) Gauge Theory with No Gauge Hierarchy Problem, Nucl. Phys. B 206 (1982) 387 [InSPIRE].

[46] J. Hisano, T. Moroi, K. Tobe and T. Yanagida, Suppression of proton decay in the missing partner model for supersymmetric SU(5) GUT, Phys. Lett. B 342 (1995) 138 [hep-ph/9406417] [INSPIRE].

[47] B.A. Campbell, J.R. Ellis, J.S. Hagelin, D.V. Nanopoulos and R. Ticciati, Flipped SU(5) From Manifold Compactification of the Ten-dimensional Heterotic String, Phys. Lett. B 198 (1987) 200 [inSPIRE].

[48] J.L. Lopez, D.V. Nanopoulos and K.-j. Yuan, The Search for a realistic flipped SU(5) string model, Nucl. Phys. B 399 (1993) 654 [hep-th/9203025] [INSPIRE].

[49] J. Ellis, M.A.G. Garcia, N. Nagata, D.V. Nanopoulos and K.A. Olive, Starobinsky-like Inflation, Supercosmology and Neutrino Masses in No-Scale Flipped SU(5), JCAP 07 (2017) 006 [arXiv: 1704.07331] [INSPIRE].

[50] J. Ellis, M.A.G. Garcia, N. Nagata, D.V. Nanopoulos and K.A. Olive, Symmetry Breaking and Reheating after Inflation in No-Scale Flipped SU(5), JCAP 04 (2019) 009 [arXiv: 1812.08184] [INSPIRE].

[51] J. Ellis, M.A.G. Garcia, N. Nagata, D.V. Nanopoulos and K.A. Olive, Cosmology with a master coupling in flipped $\mathrm{SU}(5) \times \mathrm{U}(1)$ : the $\lambda_{6}$ universe, Phys. Lett. B 797 (2019) 134864 [arXiv: 1906. 08483] [INSPIRE]. 
[52] J. Ellis, M.A.G. Garcia, N. Nagata, D.V. Nanopoulos and K.A. Olive, Superstring-Inspired Particle Cosmology: Inflation, Neutrino Masses, Leptogenesis, Dark Matter 8 the SUSY Scale, JCAP 01 (2020) 035 [arXiv:1910.11755] [INSPIRE].

[53] E. Witten, Dimensional Reduction of Superstring Models, Phys. Lett. 155B (1985) 151 [INSPIRE].

[54] E. Cremmer, S. Ferrara, C. Kounnas and D.V. Nanopoulos, Naturally Vanishing Cosmological Constant in $N=1$ Supergravity, Phys. Lett. 133B (1983) 61 [INSPIRE].

[55] A.B. Lahanas and D.V. Nanopoulos, The Road to No Scale Supergravity, Phys. Rept. 145 (1987) 1 [INSPIRE].

[56] J.R. Ellis, C. Kounnas and D.V. Nanopoulos, No Scale Supersymmetric Guts, Nucl. Phys. B 247 (1984) 373 [INSPIRE].

[57] J.R. Ellis, J.S. Hagelin, S. Kelley and D.V. Nanopoulos, Aspects of the Flipped Unification of Strong, Weak and Electromagnetic Interactions, Nucl. Phys. B 311 (1988) 1 [INSPIRE].

[58] V. Lucas and S. Raby, Nucleon decay in a realistic $\mathrm{SO}(10)$ SUSY GUT, Phys. Rev. D 55 (1997) 6986 [hep-ph/9610293] [INSPIRE].

[59] K.S. Babu, J.C. Pati and F. Wilczek, Suggested new modes in supersymmetric proton decay, Phys. Lett. B 423 (1998) 337 [hep-ph/9712307] [INSPIRE].

[60] K.S. Babu and M.J. Strassler, A Reexamination of proton decay in supersymmetric grand unified theories, hep-ph/9808447 [INSPIRE].

[61] K.S. Babu, J.C. Pati and F. Wilczek, Fermion masses, neutrino oscillations and proton decay in the light of Super-Kamiokande, Nucl. Phys. B 566 (2000) 33 [hep-ph/9812538] [INSPIRE].

[62] N. Maekawa and Y. Muramatsu, Nucleon decay via dimension-6 operators in anomalous $\mathrm{U}(1)_{A}$ supersymmetric GUT, Phys. Rev. D 88 (2013) 095008 [arXiv: 1307.7529] [INSPIRE].

[63] N. Maekawa and Y. Muramatsu, Nucleon decay via dimension-6 operators in $E_{6} \times \mathrm{SU}(2)_{F} \times \mathrm{U}(1)_{A} S U S Y$ GUT model, PTEP 2014 (2014) 113B03 [arXiv:1401.2633] [INSPIRE].

[64] N. Maekawa and Y. Muramatsu, Flavor changing nucleon decay, Phys. Lett. B 767 (2017) 398 [arXiv: 1601.04789] [INSPIRE].

[65] W. Buchmüller and K.M. Patel, Proton decay in flux compactifications, JHEP 05 (2019) 196 [arXiv: 1904.08810] [INSPIRE].

[66] N. Okada, D. Raut and Q. Shafi, Inflation, proton decay and Higgs-portal dark matter in $\mathrm{SO}(10) \times \mathrm{U}(1)_{\psi}$, Eur. Phys. J. C 79 (2019) 1036 [arXiv:1906.06869] [INSPIRE].

[67] N. Haba, Y. Mimura and T. Yamada, Enhanced $\Gamma\left(p \rightarrow K^{0} \mu^{+}\right) / \Gamma\left(p \rightarrow K^{+} \bar{\nu}_{\mu}\right)$ as a Signature of Minimal Renormalizable SUSY SO(10) GUT, arXiv:2002.11413 [INSPIRE].

[68] J.R. Ellis, J.L. Lopez, D.V. Nanopoulos and K.A. Olive, Flipped angles and phases: A Systematic study, Phys. Lett. B 308 (1993) 70 [hep-ph/9303307] [INSPIRE].

[69] J.R. Ellis, J.L. Lopez and D.V. Nanopoulos, Lowering $\alpha_{s}$ by flipping SU(5), Phys. Lett. B 371 (1996) 65 [hep-ph/9510246] [INSPIRE].

[70] J.R. Ellis, D.V. Nanopoulos and J. Walker, Flipping SU(5) out of trouble, Phys. Lett. B $\mathbf{5 5 0}$ (2002) 99 [hep-ph/0205336] [INSPIRE].

[71] I. Dorsner and P. Fileviez Perez, Distinguishing between SU(5) and flipped SU(5), Phys. Lett. B 605 (2005) 391 [hep-ph/0409095] [INSPIRE]. 
[72] M.U. Rehman, Q. Shafi and J.R. Wickman, Minimal Supersymmetric Hybrid Inflation, Flipped SU(5) and Proton Decay, Phys. Lett. B 688 (2010) 75 [arXiv:0912.4737] [INSPIRE].

[73] T. Li, D.V. Nanopoulos and J.W. Walker, Elements of F-ast Proton Decay, Nucl. Phys. B 846 (2011) 43 [arXiv: 1003.2570] [INSPIRE].

[74] M.U. Rehman, Q. Shafi and U. Zubair, Gravity waves and proton decay in a flipped SU(5) hybrid inflation model, Phys. Rev. D 97 (2018) 123522 [arXiv: 1804.02493] [INSPIRE].

[75] A.A. Starobinsky, A New Type of Isotropic Cosmological Models Without Singularity, Phys. Lett. 91B (1980) 99 [INSPIRE].

[76] J. Ellis, D.V. Nanopoulos and K.A. Olive, No-Scale Supergravity Realization of the Starobinsky Model of Inflation, Phys. Rev. Lett. 111 (2013) 111301 [Erratum ibid. 111 (2013) 129902] [arXiv: 1305.1247] [INSPIRE].

[77] Planck collaboration, Planck 2018 results. VI. Cosmological parameters, arXiv: 1807.06209 [INSPIRE].

[78] CMB-S4 collaboration, CMB-S4 Science Book, First Edition, arXiv:1610.02743 [INSPIRE].

[79] K. Abazajian et al., CMB-S4 Science Case, Reference Design and Project Plan, arXiv: 1907.04473 [INSPIRE].

[80] M. Hazumi et al., LiteBIRD: A Satellite for the Studies of B-Mode Polarization and Inflation from Cosmic Background Radiation Detection, J. Low. Temp. Phys. 194 (2019) 443 [INSPIRE].

[81] J. Ellis, D.V. Nanopoulos and K.A. Olive, A no-scale supergravity framework for sub-Planckian physics, Phys. Rev. D 89 (2014) 043502 [arXiv:1310.4770] [INSPIRE].

[82] M. Fukugita and T. Yanagida, Baryogenesis Without Grand Unification, Phys. Lett. B 174 (1986) 45 [INSPIRE].

[83] Particle Data Group collaboration, Review of Particle Physics, Phys. Rev. D 98 (2018) 030001 [INSPIRE].

[84] P. Minkowski, $\mu \rightarrow$ er at a Rate of One Out of $10^{9}$ Muon Decays?, Phys. Lett. 67B (1977) 421 [INSPIRE].

[85] T. Yanagida, Horizontal gauge symmetry and masses of neutrinos, Conf. Proc. C $\mathbf{7 9 0 2 1 3 1}$ (1979) 95 [INSPIRE].

[86] M. Gell-Mann, P. Ramond and R. Slansky, Complex Spinors and Unified Theories, Conf. Proc. C 790927 (1979) 315 [arXiv: 1306 .4669] [InSPIRE].

[87] S.L. Glashow, The Future of Elementary Particle Physics, NATO Sci. Ser. B 59 (1980) 687 [INSPIRE].

[88] R.N. Mohapatra and G. Senjanović, Neutrino Mass and Spontaneous Parity Nonconservation, Phys. Rev. Lett. 44 (1980) 912 [INSPIRE].

[89] R.N. Mohapatra and G. Senjanović, Neutrino Masses and Mixings in Gauge Models with Spontaneous Parity Violation, Phys. Rev. D 23 (1981) 165 [inSPIRE].

[90] J. Schechter and J.W.F. Valle, Neutrino Masses in $\mathrm{SU}(2) \times \mathrm{U}(1)$ Theories, Phys. Rev. D 22 (1980) 2227 [INSPIRE].

[91] J. Schechter and J.W.F. Valle, Neutrino Decay and Spontaneous Violation of Lepton Number, Phys. Rev. D 25 (1982) 774 [InSPIRE]. 
[92] H. Georgi and D.V. Nanopoulos, Ordinary Predictions from Grand Principles: T Quark Mass in O(10), Nucl. Phys. B 155 (1979) 52 [INSPIRE].

[93] J. Hisano, D. Kobayashi, Y. Muramatsu and N. Nagata, Two-loop Renormalization Factors of Dimension-six Proton Decay Operators in the Supersymmetric Standard Models, Phys. Lett. B 724 (2013) 283 [arXiv:1302.2194] [INSPIRE].

[94] C. Muñoz, Enhancement Factors for Supersymmetric Proton Decay in $\mathrm{SU}(5)$ and $\mathrm{SO}(10)$ With Superfield Techniques, Phys. Lett. B 177 (1986) 55 [INSPIRE].

[95] L.F. Abbott and M.B. Wise, The Effective Hamiltonian for Nucleon Decay, Phys. Rev. D 22 (1980) 2208 [INSPIRE].

[96] T. Nihei and J. Arafune, The Two loop long range effect on the proton decay effective Lagrangian, Prog. Theor. Phys. 93 (1995) 665 [hep-ph/9412325] [INSPIRE].

[97] Y. Aoki, T. Izubuchi, E. Shintani and A. Soni, Improved lattice computation of proton decay matrix elements, Phys. Rev. D 96 (2017) 014506 [arXiv:1705.01338] [INSPIRE].

[98] J. Hisano, H. Murayama and T. Yanagida, Nucleon decay in the minimal supersymmetric SU(5) grand unification, Nucl. Phys. B 402 (1993) 46 [hep-ph/9207279] [INSPIRE].

[99] J. Hisano, D. Kobayashi and N. Nagata, Enhancement of Proton Decay Rates in Supersymmetric SU(5) Grand Unified Models, Phys. Lett. B 716 (2012) 406 [arXiv: 1204.6274] [INSPIRE].

[100] J.R. Ellis, M.K. Gaillard and D.V. Nanopoulos, On the Effective Lagrangian for Baryon Decay, Phys. Lett. 88B (1979) 320 [inSPIRE].

[101] I. Esteban, M.C. Gonzalez-Garcia, A. Hernandez-Cabezudo, M. Maltoni and T. Schwetz, Global analysis of three-flavour neutrino oscillations: synergies and tensions in the determination of $\theta_{23}, \delta_{C P}$ and the mass ordering, JHEP 01 (2019) 106 [arXiv: 1811.05487] [INSPIRE].

[102] http://www.nu-fit.org.

[103] A. Takenaka, Search for Proton Decay: The Latest Results from Super-Kamiokande, talk at NuPhys2019, Cavendish Conference Centre, London, U.K., 18 December 2019 [https://indico.cern.ch/event/818781/contributions/3668031/attachments/1964245/ 3266410/PDK.NuPhys2019.v4.pdf].

[104] Super-Kamiokande collaboration, Search for proton decay via $p \rightarrow e^{+} \pi^{0}$ and $p \rightarrow \mu^{+} \pi^{0}$ in 0.31 megaton.years exposure of the Super-Kamiokande water Cherenkov detector, Phys. Rev. D 95 (2017) 012004 [arXiv: 1610.03597] [InSPIRE].

[105] Super-Kamiokande collaboration, Search for Nucleon Decay via $n \rightarrow \bar{\nu} \pi^{0}$ and $p \rightarrow \bar{\nu} \pi^{+}$ in Super-Kamiokande, Phys. Rev. Lett. 113 (2014) 121802 [arXiv:1305.4391] [INSPIRE].

[106] SuPER-KAmioKANDE collaboration, Search for nucleon decay into charged antilepton plus meson in 0.316 megaton-years exposure of the Super-Kamiokande water Cherenkov detector, Phys. Rev. D 96 (2017) 012003 [arXiv:1705.07221] [InSPIRE]. 